\title{
A Method to Identify the Incomplete Framework of Discernment in Evidence Theory
}

\author{
Wen Jiang, Yue Chang, and Shiyu Wang \\ School of Electronics and Information, Northwestern Polytechnical University, Xian, Shaanxi 710072, China \\ Correspondence should be addressed to Wen Jiang; jiangwen@nwpu.edu.cn
}

Received 30 July 2017; Revised 16 October 2017; Accepted 5 November 2017; Published 10 December 2017

Academic Editor: Anna M. Gil-Lafuente

Copyright (c) 2017 Wen Jiang et al. This is an open access article distributed under the Creative Commons Attribution License, which permits unrestricted use, distribution, and reproduction in any medium, provided the original work is properly cited.

\begin{abstract}
Dempster-Shafer evidence theory is a very useful tool in dealing with the decision-making of uncertain information. However, the classical evidence theory is no longer applicable when the frame of discernment (FOD) is incomplete. Moreover, incomplete FOD is an important reason for the emergence of conflict. So it is necessary to identify whether the FOD of the system is complete or not. In this paper, a method is proposed to identify the incomplete FOD under framework of the generalized evidence theory dealing with incomplete information. Within the proposed method, pieces of evidence are generated from the attributes of each sample firstly; then three criteria are used to identify weather the FOD is incomplete according to these evidence. The main parameters of the criteria are the amount of $\emptyset$ being a focal element in generated evidence, the mass of $\emptyset$ in the weighted average evidence from generated evidence, and the mass of $\emptyset$ in the combination of generated evidence. Some experiments are used to demonstrate the effectiveness of the proposed method.
\end{abstract}

\section{Introduction}

Dempster-Shafer evidence theory (D-S evidence theory) [1, 2] is widely used in many fields such as decision-making [3$6]$, evidential reasoning $[7,8]$, uncertainty measure $[9,10]$, and others [11-15] because of its advantages in handling uncertainty information. This theory is also widely used in practical applications, such as fault diagnosis [16, 17], knowledge acquisition [18], risk and reliability analysis [19, 20], and failure mode [21-23]. While the counterintuitive results can be obtained when the given evidences highly conflict with each other, hundreds of methods have been proposed to address this issue [24]. In D-S evidence theory, conflict management is still an important issue. In general, there are two main reasons that may lead to conflict: one is the incomplete frame of discernment (FOD) and the other is that the sensors are disturbed. In order to better implement the combination of conflicting evidence, it is necessary and important to identify whether the FOD is complete or not.

In previous studies, Lefevre et al. [25] proposed a unified belief function combination method to manage the conflict, mainly considering the issue of conflict redistribution. Haenni's view is to get the pretreatment evidence and then use Dempster combination rule to manage the conflict [26]. Murphy [27] presented a method to combine belief functions named averaging to balance multiple evidence. But it does not offer convergence toward certainty. Based on this, an improved method is presented in [28]. While these studies ignored the fact that the incomplete FOD is also an important cause that may lead to the conflict, for that, Smets and Kennes [29] proposed the TBM model under the open world assumption. Recently, a generalized evidence theory was presented in [30], addressing the combination of conflicting evidence in open world. It greatly expands the application of evidence theory like fuzzy [31-33] and game theory [34] and jumps out of the original model in dealing with conflicts. But the research aforementioned also did not tell under which condition the system is in incomplete FOD. According to previous studies, the research on identifying the incomplete FOD is still an open issue and has not been given deserved attention.

In this paper, a method is proposed to identify the incomplete FOD under framework of the generalized evidence theory. Generalized evidence theory [30] is a novel theory which can express and deal with uncertain information in an incomplete FOD. In consideration of empty set $\emptyset$ can express 
the information of incomplete FOD; three parameters of $\emptyset$ are used in the proposed method. They are the mass of $\emptyset$ in the weight average evidence from generated pieces of evidence, the amount of $\emptyset$ being a focal element in generated pieces of evidence, and the mass of $\emptyset$ in the combination of generated pieces of evidence. Within the proposed method, pieces of evidence can be generated from the attributes of each sample firstly; then three criteria are used to identify weather the FOD is incomplete according to these evidence.

In this paper, a method to identify the incomplete FOD, which takes into consideration the information in both the evidence and the samples, is proposed. The method uses the correlation coefficient $r_{\mathrm{BPA}}$ [35] which has a better performance compared with other coefficients to express the similarity of evidence. From three aspects the proposed method collects the information about the FOD of the system, respectively, the mass of $\emptyset$ in one piece of evidence, the mass distribution of $\emptyset$ in weighted average evidence, and the mass distribution of $\emptyset$ in combination result. Some experiments are used to demonstrate the effectiveness of the proposed method. The experiments show that, for a collected sample, if the criteria can be satisfied, the system is seen incomplete. If it is not satisfied, it is seen as a complete FOD.

The rest of this paper is organised as follows. In Section 2, the preliminaries about D-S evidence and generalized evidence theory are briefly introduced. Section 3 presents the proposed method with three criteria. In Section 4, some experiments are shown to demonstrate the effectiveness of our method. An application about the motor rotor fault diagnosis is shown in Section 5. Finally, a brief conclusion is made in Section 6.

\section{Preliminaries}

2.1. Dempster-Shafer (D-S) Evidence Theory. D-S evidence theory is introduced by Dempster [1] and then developed by Shafer [2]. Owing to its outstanding performance in uncertainty model and process, this theory is widely applied to decision-making, optimization and reliability, and risk analysis.

Definition 1. Let $\Theta$ be a finite nonempty set of mutually exclusive hypotheses, indicated by

$$
\Theta=\left\{\theta_{1}, \theta_{2}, \ldots, \theta_{i}, \ldots, \theta_{N}\right\},
$$

where set $\Theta$ is called a frame of discernment. The power set of $\Theta, 2^{\Theta}$, is indicated as follows:

$$
2^{\Theta}=\left\{\emptyset,\left\{\theta_{1}\right\}, \ldots,\left\{\theta_{N}\right\},\left\{\theta_{1}, \theta_{2}\right\}, \ldots,\left\{\theta_{1}, \theta_{2}, \ldots, \theta_{i}\right\}, \ldots, \Theta\right\} .
$$

Definition 2. A mass function is a mapping $m$ from $2^{\Theta}$ to $[0,1]$, formally noted by

$$
m: 2^{\Theta} \longrightarrow[0,1]
$$

which satisfies the following condition:

$$
\begin{aligned}
m(\emptyset) & =0, \\
\sum_{A \in 2^{\Theta}} m(A) & =1 .
\end{aligned}
$$

When $m(A)>0, A$ is called a focal element of the mass function.

Definition 3. Evidence combination in D-S evidence theory is noted as $\oplus$. Assume that there are two BPAs indicated by $m_{1}$ and $m_{2}$; the evidence combination of the two BPAs with Dempster's combination rule [1] is formulated as follows:

$$
m(A)= \begin{cases}0 & A=\emptyset, \\ \frac{1}{1-K} \sum_{B \cap C=A} m_{1}(B) m_{2}(C) & A \neq \emptyset,\end{cases}
$$

where

$$
K=\sum_{B \cap C=\emptyset} m_{1}(B) m_{2}(C) .
$$

$K$ reflects the conflict between the two BPAs $m_{1}$ and $m_{2}$.

When given $n(n>2)$ pieces of evidence, the evidence fusion with Dempster's combination rule can be shown in (8). It attributes to the merit of the commutativity and associativity of the combination rule

$$
m=m_{1} \oplus m_{2} \oplus \cdots \oplus m_{n}=\left(\left(\left(m_{1} \oplus m_{2}\right) \oplus \cdots\right) \oplus m_{n}\right) .
$$

Recently, Jiang proposed a correlation coefficient [35] to measure the degree of evidence.

Definition 4. For a discernment frame $\Theta$ with $N$ elements, suppose the mass of two pieces of evidence denoted by $m_{1}, m_{2}$. A correlation coefficient is defined as follows:

$$
r_{\mathrm{BPA}}\left(m_{1}, m_{2}\right)=\frac{c\left(m_{1}, m_{2}\right)}{\sqrt{c\left(m_{1}, m_{1}\right) c\left(m_{2}, m_{2}\right)}},
$$

where $c\left(m_{1}, m_{2}\right)$ is degree of correlation denoted as

$$
c\left(m_{1}, m_{2}\right)=\sum_{i=1}^{2^{N}} \sum_{j=1}^{2^{N}} m_{1}\left(A_{i}\right) m_{2}\left(A_{j}\right) \frac{\left|A_{i} \cap A_{j}\right|}{\left|A_{i} \cup A_{j}\right|},
$$

where $i, j=1,2, \ldots, 2^{n} ; A_{i}, A_{j}$ is the focal elements of mass, respectively; and $|\cdot|$ is the cardinality of subset.

2.2. Generalized Evidence Theory. Generalized evidence theory (GET) [30] eliminates the constraint close world and builds framework on an open world. To some extent, GET is the extension of the D-S theory and can express and deal with more uncertain information in the open world, comparing with D-S theory.

Definition 5. Let $U$ be a frame of discernment (FOD) in an open world. Its power set $2_{G}^{U}$ is composed of $2^{|U|}$ propositions. For $\forall A \subset U$, the mass function is a mapping $m: 2_{G}^{U} \rightarrow[0,1]$ that satisfies

$$
\sum_{A \subseteq 2_{G}^{U}} m_{G}(A)=1
$$

then $m_{G}$ is the generalized basic probability assignment (GBPA) of the FOD $U$. 
The difference between GBPA and classical BPA is the restriction of (4), which means the empty set should also be regarded as a focal element and represents the union of the focal element out of the given FOD. And if $m(\emptyset)=0$, the GBPA degenerates to a classical BPA.

The same as GET, TBM model also assigns mass to empty set to represent unknown information. But the difference is the generation process of the mass of empty set. TBM model is simply to remove the normalization process of the Dempster's combination rule and assigns the value of conflict coefficient $K$ to empty set. When generating evidence, the mass of empty set is still 0 , while in GET, when generating evidence, mass can be assigned to empty set. This means there is no the restriction of $m(\emptyset)=0(4)$.

Definition 6. Given two GBPAs $\left(m_{1}\right.$ and $\left.m_{2}\right), m_{1}(\emptyset)$ and $m_{2}(\emptyset)$ are regarded as conflict with each other and the mass distribution of $m(\emptyset)$ should be assigned to conflict. The generalized combination rule (GCR) is defined as follows:

$$
\begin{aligned}
m(A) & =\frac{(1-m(\emptyset)) \sum_{B \cap C=A} m_{1}(B) m_{2}(C)}{1-K}, \\
K & =\sum_{B \cap C=\emptyset} m_{1}(B) m_{2}(C), \\
m(\emptyset) & =m_{1}(\emptyset) m_{2}(\emptyset),
\end{aligned}
$$

with

$$
m(\emptyset)=1 \quad \text { iff } K=1 .
$$

Equation (13) defines the generalized conflict coefficient, and when $m(\emptyset)=0$ means the framework of discernment is complete, the generalized conflict coefficient degenerates to a classical conflict coefficient.

While Jiang and Zhan pointed that there are two shortcomings of GCR in [30], one is the way to obtain that $m(\emptyset)$ is unreasonable and lacks specific physical meaning. The other is that the way to obtain generalized conflict coefficient $K$ in (16) is not consistent with the GBPA. So the modified generalized combination rule (mGCR) in GET was proposed in [30].

Definition 7. In mGCR, $m_{1}(\emptyset)$ and $m_{2}(\emptyset)$ are considered as a support for $\emptyset$. The orthogonal sum of $m_{1}(\emptyset)$ and $m_{2}(\emptyset)$ should also be normalized like other focal elements. Given two GBPAs $\left(m_{1}\right.$ and $\left.m_{2}\right)$, the mGCR is defined as follows:

$$
\begin{aligned}
m(A) & =\frac{\sum_{A_{i} \cap B_{j}=A} m_{1}\left(A_{i}\right) m_{2}\left(B_{j}\right)}{1-K}, \\
m(\emptyset) & =\frac{m_{1}(\emptyset) m_{2}(\emptyset)}{1-K}
\end{aligned}
$$

with

$$
\begin{aligned}
K & =\sum_{\substack{A_{i} \cap B_{j}=\emptyset \\
A_{i} \cup B_{j} \neq \emptyset}} m_{1}\left(A_{i}\right) m_{2}\left(B_{j}\right), \\
m(\emptyset) & =1 \quad \text { if } K=1, \\
\text { or } \sum_{A \neq \emptyset} m(A) & =0 .
\end{aligned}
$$

Also the distance between two bodies of evidence based on GET is proposed the same as in D-S evidence theory.

Definition 8. Let $m_{1}$ and $m_{2}$ be the two GBPAs on the framework of discernment $U$; the distance between $m_{1}$ and $m_{2}$ can be defined as follows:

$$
d_{\mathrm{GBPA}}\left(m_{1}, m_{2}\right)=\sqrt{\frac{1}{2}\left(\vec{m}_{1}-\vec{m}_{2}\right)^{T} \bar{D}\left(\vec{m}_{1}-\vec{m}_{2}\right)},
$$

where $\bar{D}$ is an $2^{N} \times 2^{N}$ dimension matrix and its element is expressed as follows:

$$
D(A, B)=\frac{|A \cap B|}{|A \cup B|}, \quad A, B \in 2^{U}, D(\emptyset, \emptyset)=1,
$$

and its computing method is

$$
\begin{aligned}
& d_{\mathrm{GBPA}}\left(m_{1}, m_{2}\right) \\
& \quad=\sqrt{\frac{1}{2}\left(\left\|\vec{m}_{1}\right\|^{2}+\left\|\vec{m}_{2}\right\|^{2}-2\left\langle\vec{m}_{1}, \vec{m}_{2}\right\rangle\right)},
\end{aligned}
$$

where $\left\langle\vec{m}_{1}, \vec{m}_{2}\right\rangle=\sum_{i=1}^{2^{N}} \sum_{j=1}^{2^{N}} m_{1}(A) m_{2}(A)\left(\left|A_{i} \cap A_{j}\right| / \mid A_{i} \cup\right.$ $\left.A_{j} \mid\right),\left\|\vec{m}_{1}\right\|^{2}=\langle\vec{m}, \vec{m}\rangle$.

Equation (19) can be used in the situation when the frame of discernment is complete, and the result is similar to the distance using the definition in [36].

\section{The Proposed Method}

Generally speaking, the empty set $\emptyset$ indicates that no elements are included. In the classical evidence theory, there is no mass assignment to $\emptyset$. While in GET [30], it is considered to indicate the elements that are not in the framework, which presents the information that is out of the FOD. Based on this idea, a method mainly employing the mass of $\emptyset$ is proposed under the GET framework to identify the incomplete FOD.

An incomplete FOD means that there are targets or classes or anything else that are not included in the current FOD. Let us consider a classification problem. Assume it is known that all known samples belong to $n$ classes $c_{1}, c_{2}, \ldots, c_{n}$ which constitute a FOD $\Theta=\left\{c_{1}, c_{2}, \ldots, c_{n}\right\}$. And it is assumed that each sample has $q$ attributes. Now a new sample $T$ is obtained. How can we identify the completeness of FOD $\Theta$ according to $T$ ? In this paper, for the sample $T$, at first $q$ GBPAs which allow the empty set to have mass (i.e., $m(\emptyset)>$ 0 ), denoted as $m_{1}, m_{2}, \ldots, m_{q}$, will be generated from the $q$ attributes. Then, three criteria are used as follows. 


\subsection{Criterion 1}

Criterion 1. Let $r_{i}$ be a variable taking value in $\{0,1\}, i=$ $1, \ldots, q$. If $m_{i}(\emptyset)>0.5$ in GBPA $m_{i}$; then $r_{i}=1$; else, $r_{i}=0$. Sample $T$ supports that FOD $\Theta$ is incomplete if $\sum_{i=1}^{q} r_{i}>q / 2$. Otherwise, FOD $\Theta$ is said to be complete.

This criterion illustrates that if the amount of the initial generated GBPA of $\emptyset$ whose value exceeds 0.5 is more than half of the quantity of evidence, it is considered in an incomplete FOD. There is a physical meaning of the criterion. Firstly, the parameter $m(\emptyset)$ of a generated GBPA represents the evidence's confidence to support the incomplete FOD. That is because $\emptyset$ is treated as a focal element which expresses the elements that are not in the FOD. That is to say if the distribution of $m(\emptyset)$ is larger, there is a larger support that the FOD is incomplete. Then assuming 0.5 is a threshold, if $m(\emptyset)$ exceeds the threshold, this evidence is judged supporting an incomplete FOD. Therefore, the amount of evidence which satisfies $m_{i}(\emptyset)>0.5$, equivalent to $\sum_{i=1}^{q} r_{i}$, is used to identify the FOD. If the criteria can be satisfied, which says more than half of the evidence support $T$ is out of the FOD, the FOD is incomplete.

\subsection{Criterion 2}

Criterion 2. Let $\bar{m}$ be the weighted average of $m_{1}, m_{2}, \ldots, m_{q}$. FOD $\Theta$ is incomplete if $\bar{m}(\emptyset)>0.5$. Otherwise, FOD $\Theta$ is said to be complete.

The criterion indicates if the mass of $\emptyset$ in weighted average evidence $\bar{m}$ is more than 0.5 , it is considered in an incomplete FOD. In this criterion, a weighted average of $m_{1}, m_{2}, \ldots, m_{q}$, namely, $\bar{m}$, is calculated by considering that the evidence generated from different attributes should have different importance. $\bar{m}(\emptyset)$ represents the total support of the incomplete FOD, taking the correlativity and difference of the generated evidence into account. That is to say the value of $\bar{m}(\emptyset)$ also is an information to identify the FOD. And if the value of $\bar{m}(\emptyset)$ is larger, there is a larger support that the FOD is incomplete. Assuming 0.5 is a threshold, if $\bar{m}(\emptyset)>0.5$, it is judged as supporting an incomplete FOD.

As shown above, this criterion is based on the weighted average evidence $\bar{m}$. In this paper, Deng's approach given in [28] is used to obtain $\bar{m}$. The process is given as below.

Step 1. For each pair of generated GBPA $m_{i}$ and $m_{j}$, the similarity between $m_{i}$ and $m_{j}$ is denoted as $\operatorname{Sim}_{i j}$. Deng proposed to calculate $\operatorname{Sim}_{i j}$ based on the distance of evidence. While Jiang discussed in [35] that the correlation coefficient she proposed has a better performance compared with distance of evidence, so in this paper the correlation coefficient is used to measure the similarity measure $\operatorname{Sim}_{i j}$.

Definition 9. Let $U$ be a frame of discernment (FOD) in an open world, containing $N$ mutually exclusive and exhaustive hypotheses. The similarity measure $\mathrm{Sim}_{i j}$ is expressed as

$$
\operatorname{Sim}_{i j}=r_{\mathrm{BPA}}\left(m_{i}, m_{j}\right)=\frac{c\left(m_{1}, m_{2}\right)}{\sqrt{c\left(m_{1}, m_{1}\right) c\left(m_{2}, m_{2}\right)}}
$$

where

$$
c\left(m_{1}, m_{2}\right)=\sum_{i=1}^{2^{N}} \sum_{j=1}^{2^{N}} m_{1}\left(A_{i}\right) m_{2}\left(A_{j}\right) \frac{\left|A_{i} \cap A_{j}\right|}{\left|A_{i} \cup A_{j}\right|},
$$

where $i, j=1,2, \ldots, 2^{N} ; A_{i}, A_{j}$ is the focal elements of mass, respectively; and $|\cdot|$ is the cardinality of subset, especially, $|\emptyset \cap \emptyset| /|\emptyset \cup \emptyset|=0$.

Step 2. For the $q$ generated GBPA, we can calculate the similarity measure $\operatorname{Sim}_{i j}$ between $m_{i}$ and $m_{j}(i, j=1, \ldots, q)$. So a similarity measure matrix (SMM) can be constructed to give the insight into the agreement between the pieces of evidence:

$$
\operatorname{SMM}=\left[\begin{array}{cccccc}
1 & \operatorname{Sim}_{12} & \cdots & \operatorname{Sim}_{1 j} & \cdots & \operatorname{Sim}_{1 q} \\
\vdots & \vdots & & \vdots & \vdots & \vdots \\
\operatorname{Sim}_{i 1} & \operatorname{Sim}_{i 2} & \cdots & \operatorname{Sim}_{i j} & \cdots & \operatorname{Sim}_{i q} \\
\vdots & \vdots & & \vdots & \vdots & \vdots \\
\operatorname{Sim}_{q 1} & \operatorname{Sim}_{q 2} & \cdots & \operatorname{Sim}_{q j} & \cdots & 1
\end{array}\right] .
$$

Step 3. After obtaining the similarity measure matrix SMM, the support degree $\sup \left(m_{i}\right)$ of each evidence $m_{i}(i, j=$ $1,2, \ldots, q)$ is defined by

$$
\sup \left(m_{i}\right)=\sum_{\substack{j=1 \\ j \neq i}}^{q} \operatorname{Sim}\left(m_{i}, m_{j}\right)
$$

Then, the credibility degree $\operatorname{Crd}_{i}$ of evidence $m_{i}$ (i.e., $i=$ $1,2, \ldots, q)$ is obtained

$$
\operatorname{Crd}_{i}=\frac{\sup \left(m_{i}\right)}{\sum_{i=1}^{q} \sup \left(m_{i}\right)} .
$$

For each piece of evidence its credibility degree is seen as its weight.

Step 4. Finally, the modified weighted average evidence $\bar{m}$ is given as

$$
\bar{m}=\sum_{i=1}^{q} \operatorname{Crd}_{i} \times m_{i}
$$

Once $\bar{m}$ is obtained, $\bar{m}(\emptyset)$ can be known as well. According to this criterion, if $\bar{m}(\emptyset)>0.5$, it can be judged that the FOD is incomplete.

\subsection{Criterion 3}

Criterion 3. Let $m_{\mathrm{mGCR}}$ be the combination of $m_{1}, m_{2}, \ldots, m_{q}$ by using the modified generalized rule (mGCR). FOD $\Theta$ is incomplete if $m_{\mathrm{mGCR}}(\emptyset)>0.5$. Otherwise, FOD $\Theta$ is said to be complete.

This criterion shows that if the mass of $\emptyset$ in the combination of generated pieces of evidence with $\mathrm{mGCR}$ is more 
than 0.5 , it is considered in an incomplete FOD. That is because the parameter $m_{\mathrm{mGCR}}(\emptyset)$ represents the pieces of evidence's total confidence of the incomplete FOD, and the combination rule takes all generated pieces of evidence into account to get a final result to identify which set the sample belongs to. If the assignment of $m_{\mathrm{mGCR}}(\emptyset)$ is large, which means the assignment of other set will be inversely small, it is supporting the set $\emptyset$ and the incomplete FOD. Assuming 0.5 is a threshold, if $m_{\mathrm{mGCR}}(\emptyset)>0.5$, this system is judged supporting the incomplete FOD.

3.4. A Numerical Example for the Three Criteria. The proposed method can be used to identify the incomplete FOD, as long as we obtain the three parameters $\sum_{i=1}^{q} r_{i}, \bar{m}(\emptyset)$, $m_{\mathrm{mGCR}}(\emptyset)$. In this subsection, an illustrative example is given to show the identification result according to the three criteria.

Example 10. Assume there is a FOD $\Theta=\{a, b\}$. A new sample with $q=4$ attributes is obtained. It is assumed that four GBPAs are generated from this sample; these GPBAs are shown as follows:

$$
\begin{aligned}
m_{1}: & m_{1}(a)=0, \\
& m_{1}(b)=0.7988, \\
& m_{1}(a, b)=0, \\
& m_{1}(\emptyset)=0.2012, \\
m_{2}: & m_{2}(a)=0, \\
& m_{2}(b)=0.4839, \\
& m_{2}(a, b)=0, \\
& m_{2}(\emptyset)=0.5161, \\
m_{3}: & m_{3}(a)=0, \\
& m_{3}(b)=0.2143, \\
& m_{3}(a, b)=0, \\
& m_{3}(\emptyset)=0.7857, \\
m_{4}: & m_{4}(a)=0, \\
& m_{4}(b)=0, \\
& m_{4}(a, b)=0, \\
& m_{4}(\emptyset)=1 .
\end{aligned}
$$

Now, we can use the proposed criteria to identify the completeness of FOD $\Theta$ in terms of $m_{1}, m_{2}, m_{3}, m_{4}$.

At first, according to Criterion 1, FOD $\Theta$ is incomplete, since $m_{2}(\emptyset)=0.5161, m_{3}(\emptyset)=0.7857, m_{4}(\emptyset)=1$ which means $\sum_{i=1}^{4} r_{i}=3>q / 2=2$.

Then, from Criterion 2, FOD $\Theta$ is also identified as incomplete; the process is shown as follows.
Steps 1 and 2. The similarity measure matrix (SMM) can be calculated with (22) and (24) firstly:

$$
\mathrm{SMM}=\left[\begin{array}{llll}
1.0000 & 0.9391 & 0.7655 & 0.6556 \\
0.9391 & 1.0000 & 0.9553 & 0.8752 \\
0.7655 & 0.9553 & 1.0000 & 0.9791 \\
0.6556 & 0.8752 & 0.9791 & 1.0000
\end{array}\right] .
$$

Step 3. Then the weights of four pieces of evidence $m_{1}, m_{2}$, $m_{3}, m_{4}$ are calculated according to (25) and (26):

$$
\begin{array}{cccc}
\mathrm{Crd}_{1} & \mathrm{Crd}_{2} & \mathrm{Crd}_{3} & \mathrm{Crd}_{4} \\
\hline 0.2290 & 0.2663 & 0.2625 & 0.2413
\end{array} .
$$

Step 4. Finally, the weighted average evidence $\bar{m}$ of the four pieces of evidence is

$$
\begin{aligned}
\bar{m}(a) & =0, \\
\bar{m}(b) & =0.3687, \\
\bar{m}(a, b) & =0, \\
\bar{m}(\emptyset) & =0.6313 .
\end{aligned}
$$

Therefore, according to Criterion 2, $\bar{m}(\emptyset)=0.6313>0.5$, which shows that the FOD is still incomplete.

At last, let us use Criterion 3 to identify the completeness of $\Theta$. By using $\mathrm{mGCR}$ to combine the four pieces of evidence, the result is

$$
\begin{aligned}
m_{\mathrm{mGCR}}(a) & =0, \\
m_{\mathrm{mGCR}}(b) & =0, \\
m_{\mathrm{mGCR}}(a, b) & =0, \\
m_{\mathrm{mGCR}}(\emptyset) & =1,
\end{aligned}
$$

where $m_{\mathrm{mGCR}}(\emptyset)=1>0.5$. According to Criterion 3, the FOD $\Theta$ is incomplete. To express intuitively, these results are all shown in Table 1 .

As shown in this example, evidence $m_{1}$ supports that this sample belongs to element $a$ with 0.7988 mass of distribution; other evidences $m_{2}, m_{3}, m_{4}$ support the sample belonging to $\emptyset$ which represents the element out of the FOD $\Theta$. If all these evidence are not disturbed, human will consider this sample is in an incomplete FOD intuitively. From Table $1, \sum_{i=1}^{4} r_{i}=$ $3>q / 2=2, \bar{m}(\emptyset)=0.6313>0.5, m_{\mathrm{mGCR}}(\emptyset)=1>0.5$; three criteria are all satisfied; this sample is in an incomplete FOD as these evidences imply.

\section{Case Study}

In this section, several experiments are given to demonstrate the effectiveness of the proposed method. Iris data set, a popular data set for classification problem, is used in modeling and simulation in these experiments. In this data set, 150 samples belong to three categories, namely, setosa (a), versicolor $(b)$, and virginica $(c)$. Each category has 50 samples. 


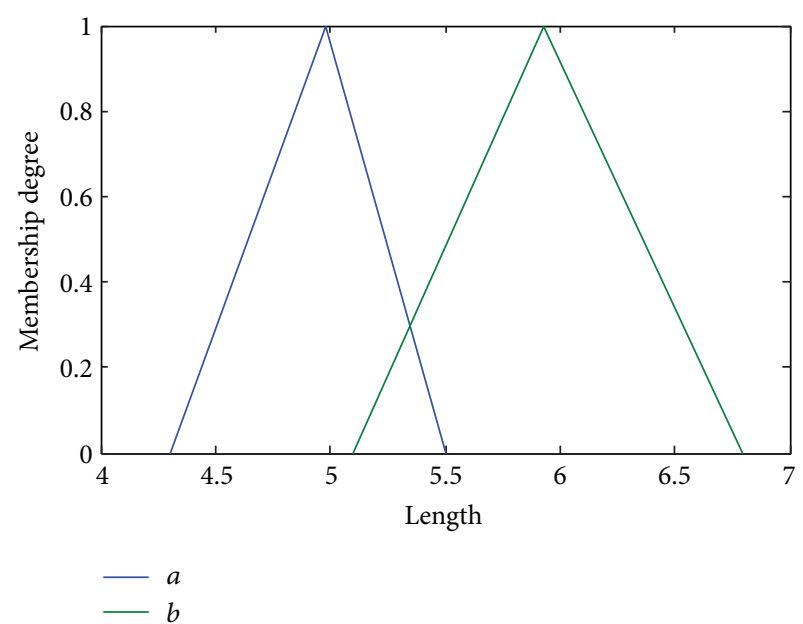

(a) SL

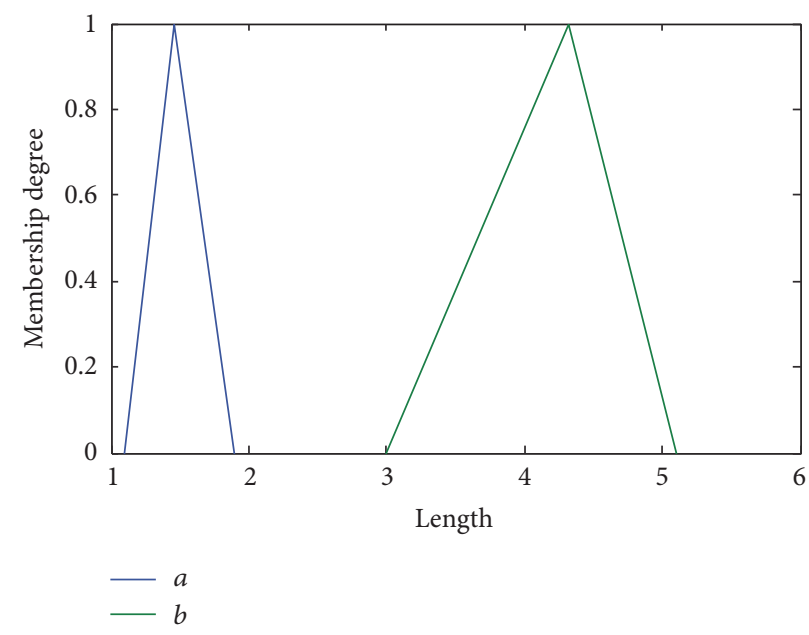

(c) PL

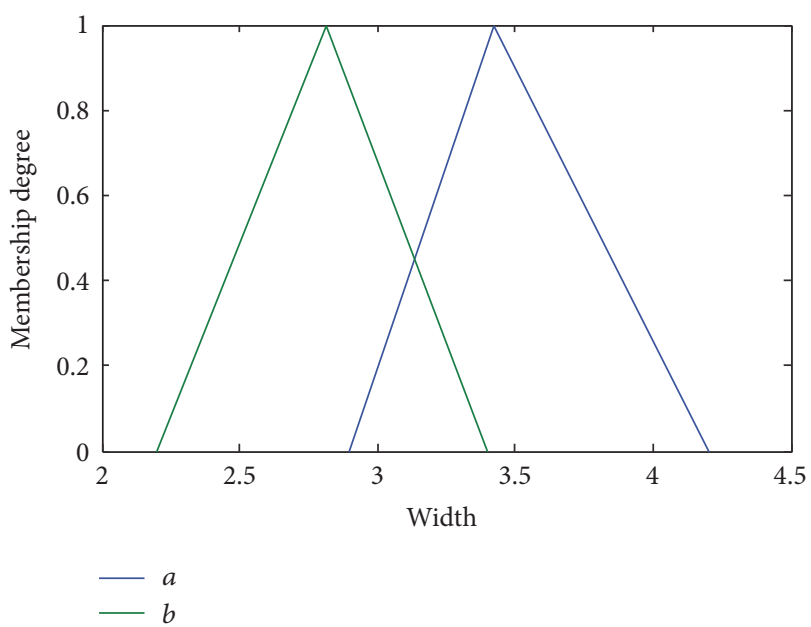

(b) SW

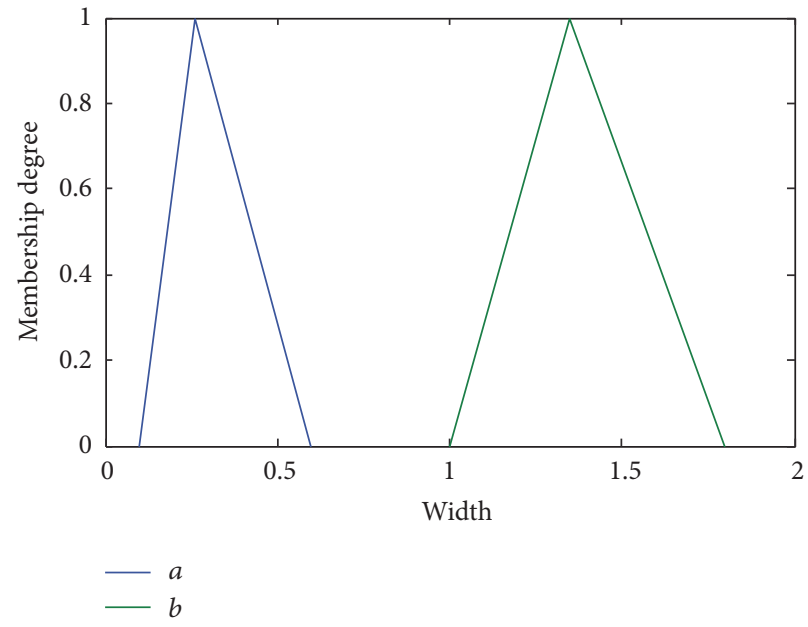

(d) PW

FIGURE 1: Training models associated with four attributes of Iris data set.

TABLE 1: The criteria parameters for Example 10.

\begin{tabular}{lccc}
\hline & $\sum_{i=1}^{4} r_{i}$ & $\bar{m}(\emptyset)$ & $m_{\mathrm{mGCR}}(\emptyset)$ \\
\hline Value & 3 & 0.6313 & 1 \\
\hline
\end{tabular}

Every sample in the data set has four attributes: sepal length $(\mathrm{PL})$, sepal width $(\mathrm{SW})$, petal length $(\mathrm{PL})$, and petal width (PW).

Now the data set is divided into two parts; one is the training set which includes 90 samples that are randomly selected from the three categories with equal quantity and the other is the test set which contains 60 samples from the three categories. In order to establish an incomplete FOD $\Theta_{a b}=\{a, b\}$, we only abstract samples belonging to categories $a$ and $b$ from the training set to construct the training model. By using the method mentioned in [37], a triangular fuzzy number model for each attribute of categories $a$ and $b$ is constituted according to the minimum, mean, and maximum value of each attribute in training set. Then for each attribute two triangular fuzzy numbers associated with categories $a$ and $b$ are generated to form a membership function for each attribute. The relevant values are listed in Table 2 and associated training modes are shown in Figure 1.

Then, based on the training models shown in Figure 1, four pieces of evidence can be generated for each sample in the test set because there are four attributes for an Iris sample. The method in [38] to generate GBPA is used in this paper. Based on the mentioned above for each sample in the test set pieces of evidence (i.e., GBPAs) associated with attributes can be obtained, as shown in Figures 2-4.

Now four experiments are carried out to verify the effectiveness of the proposed criteria of identifying incomplete FOD. Three of them will consider a single criterion, while the last one will use all three criteria simultaneously.

Experiment 1. Only consider Criterion 1 . For each sample in the test set, we can calculate $\sum_{i=1}^{4} r_{i}$. Then use Criterion 1 to judge whether FOD $\Theta_{a b}$ is incomplete or not. 
TABLE 2: Minimum, mean, and maximum value of each attribute in training models.

\begin{tabular}{lcccc}
\hline Attribute & Category & Minimum & Mean & Maximum \\
\hline \multirow{2}{*}{ SL } & $a$ & 4.3 & 5.1 & 5.8 \\
& $b$ & 4.9 & 5.9 & 7 \\
SW & $a$ & 2.0 & 2.78 & 3.4 \\
& $b$ & 2.3 & 3.5 & 4.4 \\
PL & $a$ & 1.1 & 1.44 & 1.7 \\
& $b$ & 3 & 4.3 & 5 \\
PW & $a$ & 0.1 & 0.25 & 0.6 \\
& $b$ & 1 & 1.34 & 1.7 \\
\hline
\end{tabular}

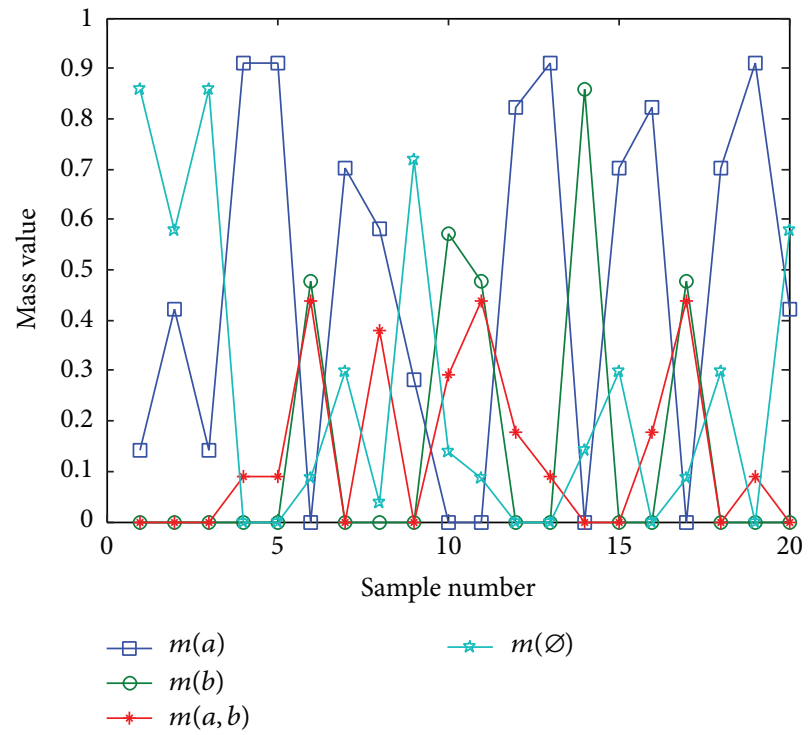

(a) SL

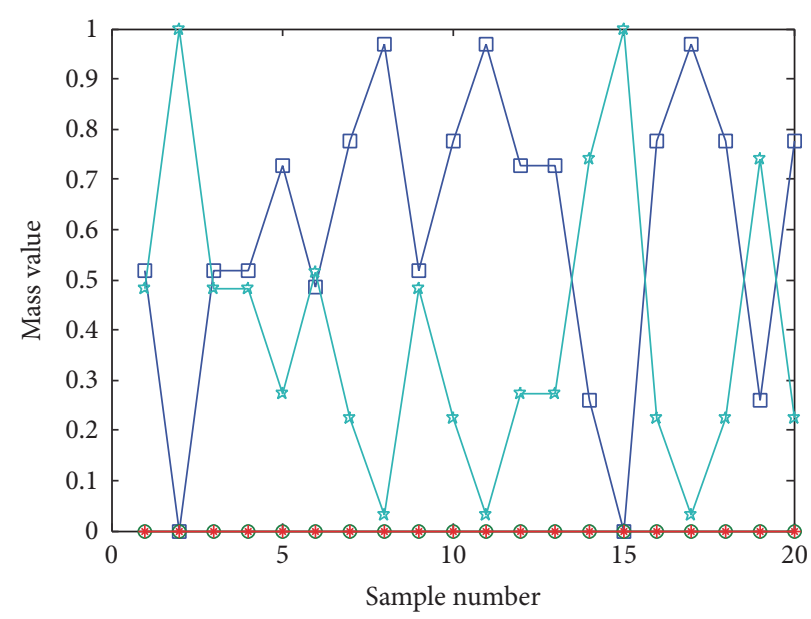

$$
\begin{aligned}
& \square m(a) \\
& -\quad m(b) \\
& -* m(a, b)
\end{aligned}
$$

$$
\rightarrow m(\varnothing)
$$

(c) PL

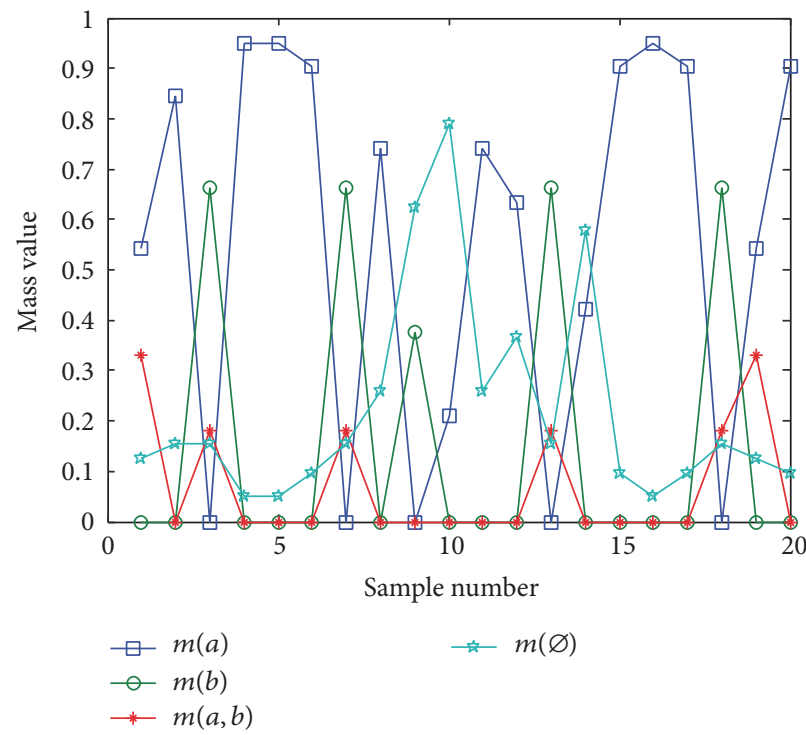

(b) SW

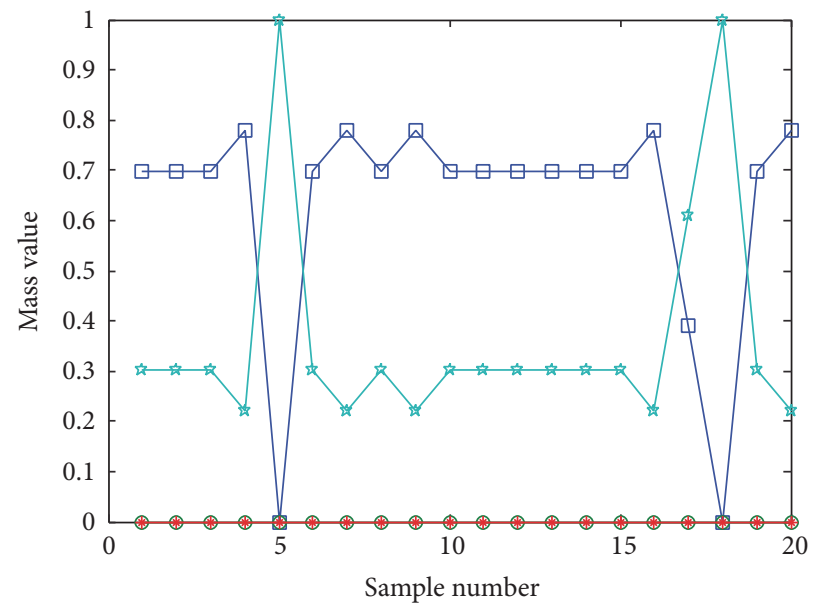

$$
\begin{aligned}
& \square m(a) \\
& -m(b) \\
& \rightarrow \quad m(a, b)
\end{aligned}
$$$$
\rightarrow m(\varnothing)
$$

(d) $\mathrm{PW}$

FIGURE 2: Generated GBPAs associated with different attributes for the samples belonging to category $a$ in the test set. 


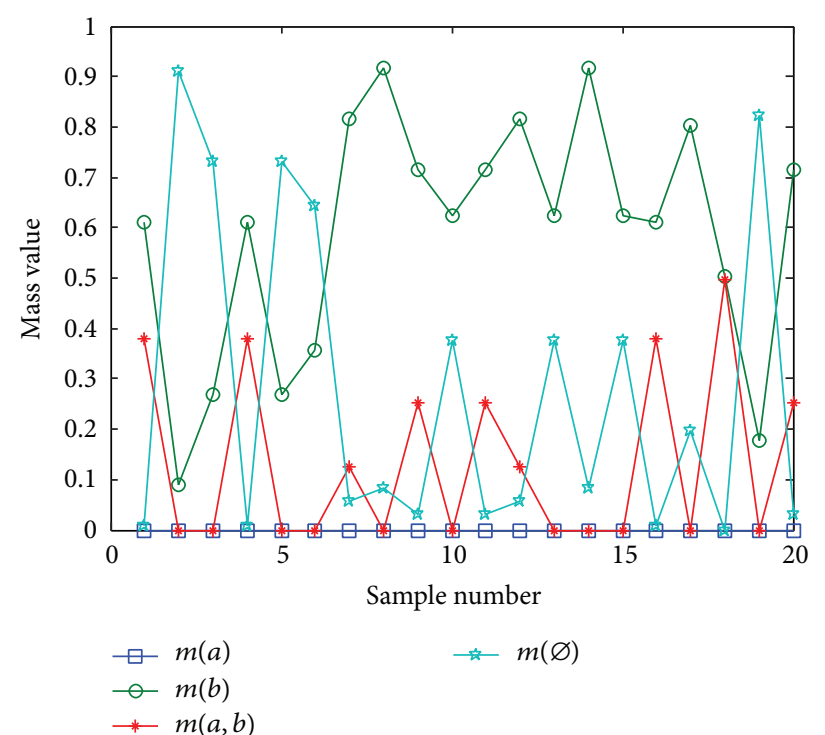

(a) SL

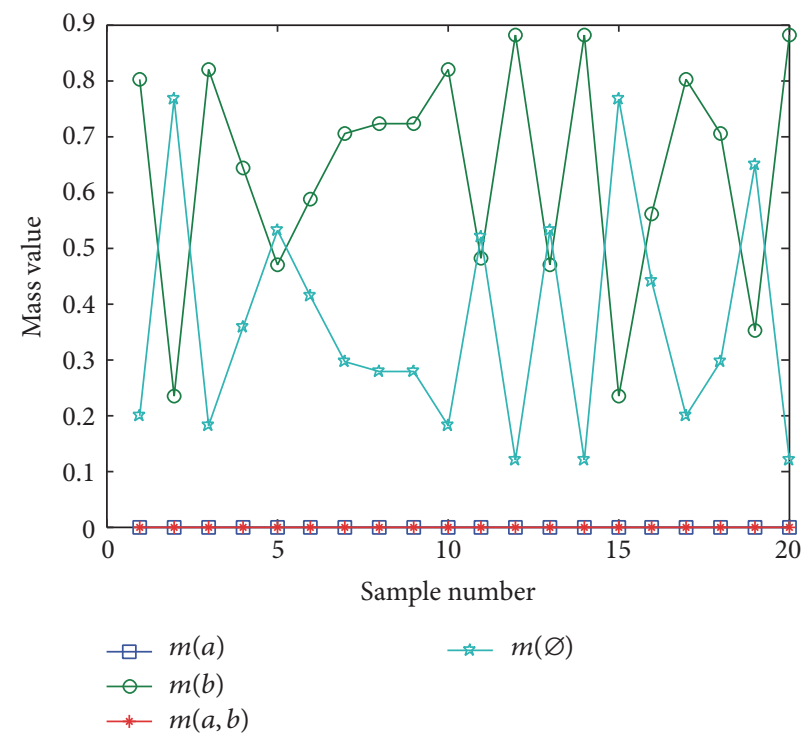

(c) PL

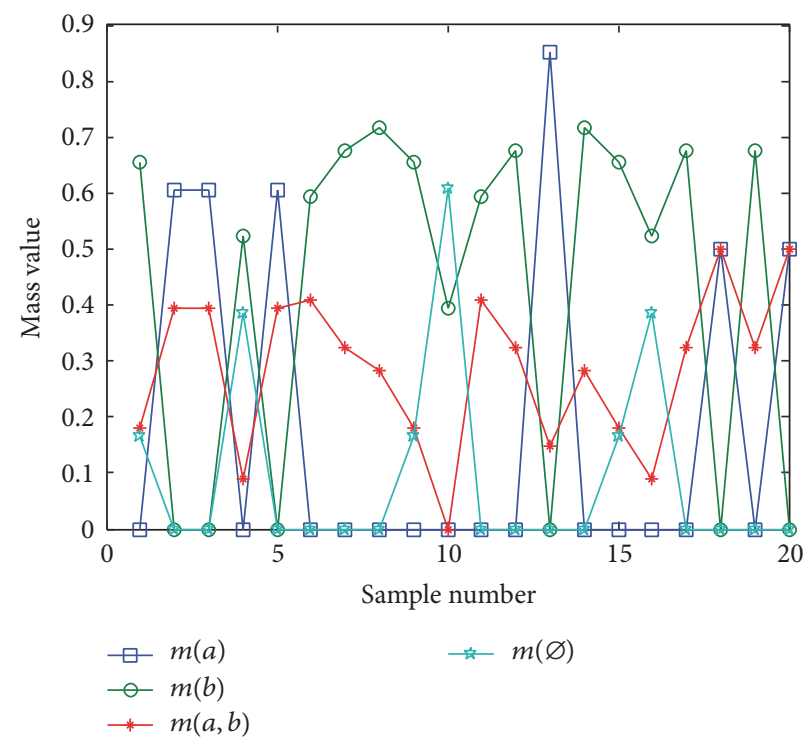

(b) SW

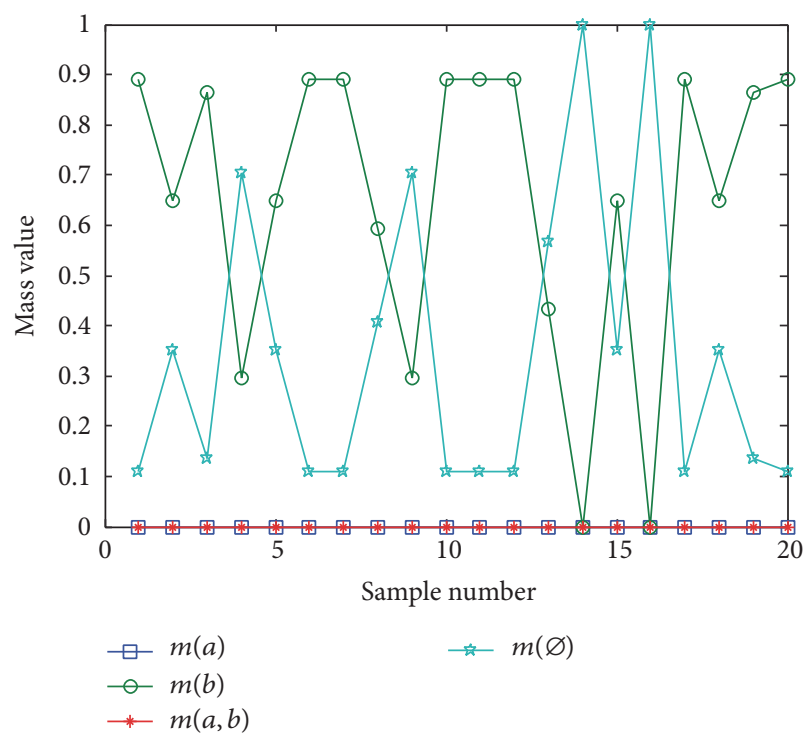

(d) PW

FIGURE 3: Generated GBPAs associated with different attributes for the samples belonging to category $b$ in the test set.

Experiment 2. Only consider Criterion 2. For the generated GBPAs of each sample in the test set, use the method proposed in Section 3.2 to derive the average weighted evidence $\bar{m}$. Then $\bar{m}(\emptyset)$ is obtained as well, according to Criterion 2, judging the completeness of FOD $\Theta_{a b}$.

Experiment 3. Only consider Criterion 3. Use mGCR to combine the generated evidence of each sample to derive $m_{\mathrm{mGCR}}$. Then $m_{\mathrm{mGCR}}(\emptyset)$ is obtained as well. According to Criterion 3 the completeness of FOD $\Theta_{a b}$ can be judged.

Experiment 4. In this case, Criterions 1, 2, and 3 are simultaneously considered. According to the method in Section 3 to derive the parameters of three criteria, judge whether the
FOD $\Theta_{a b}$ is incomplete or not. If all three criteria are satisfied, the FOD $\Theta_{a b}$ is incomplete. If one of the criteria cannot be satisfied, the FOD $\Theta_{a b}$ is complete.

In order to clearly show the results of these experiments, the confusion matrix [39] is used containing the information about actual and identified situation. Based on the matrix, some indices, for instance, accuracy, sensitivity (also called recall), and precision, have been developed to evaluate the performance of each criteria. For each sample of $a$ and $b$, if it does not meet the criteria, it means FOD $\Theta_{a b}$ is complete, which is correct. If it does, it is considered that $\Theta_{a b}$ is not complete which is incorrect, while, for each sample of $c$, it is recognized correctly only when the result supports that $\Theta_{a b}$ 


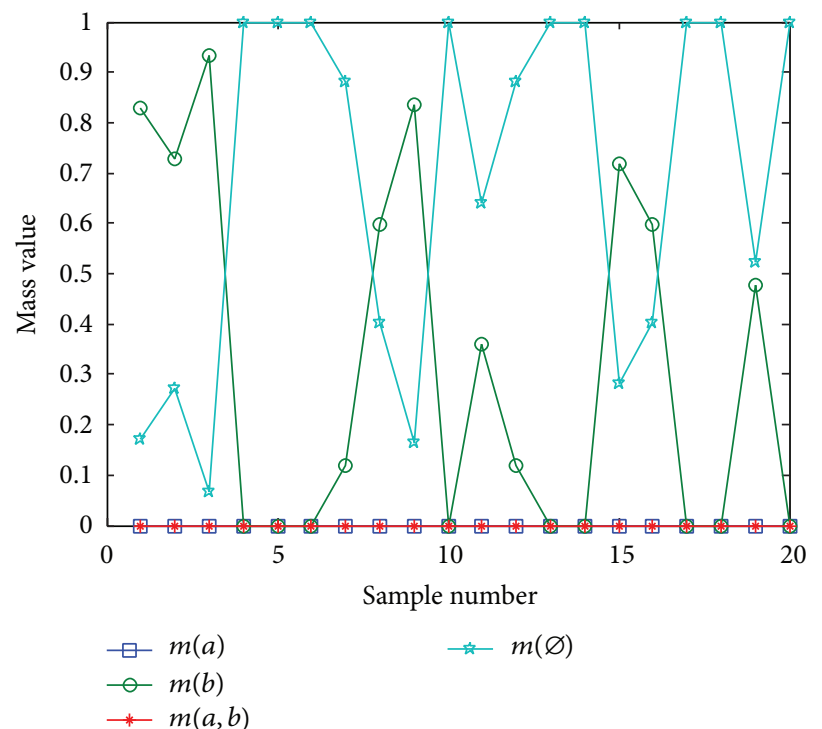

(a) SL

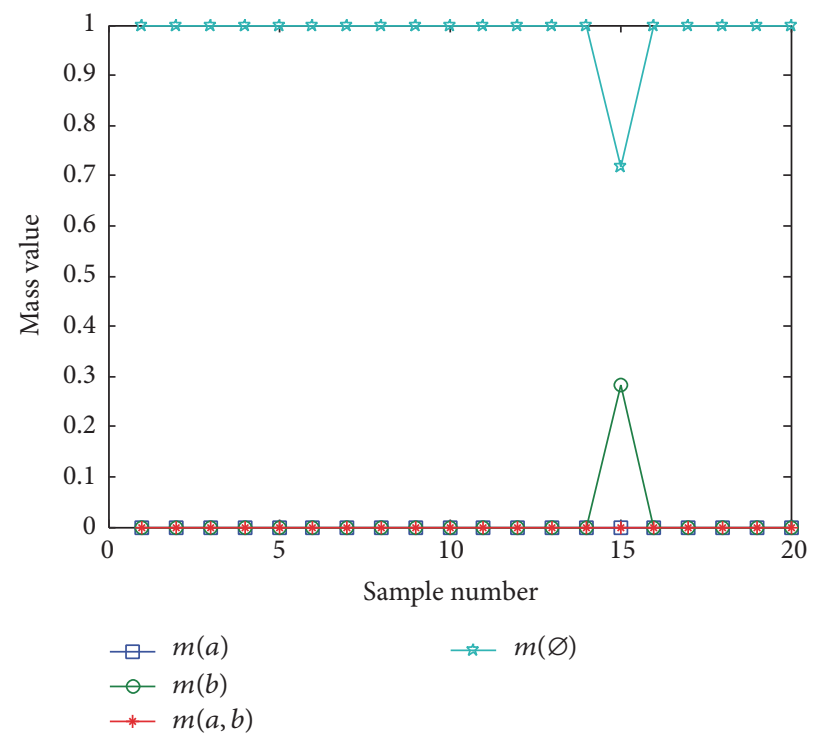

(c) PL

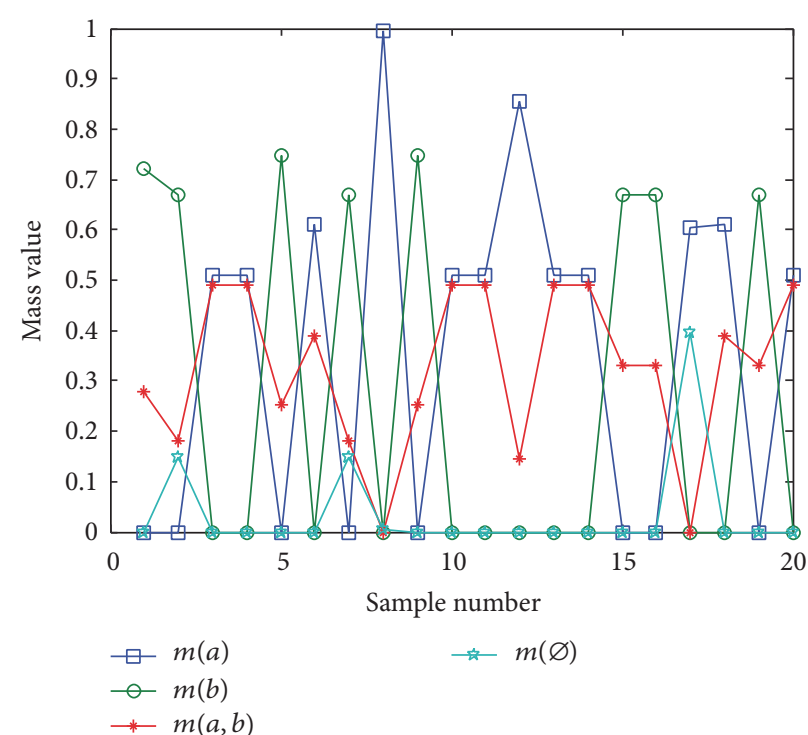

(b) SW

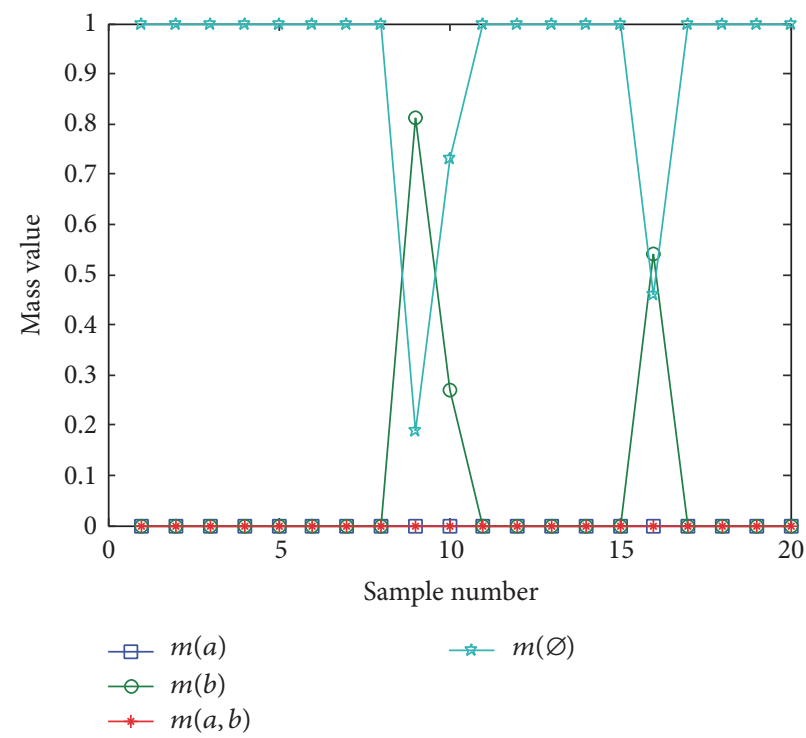

(d) PW

FIGURE 4: Generated GBPAs associated with different attributes for the samples belonging to category $c$ in the test set.

TABLE 3: The quantity of correct identification in the experiments sorted by categories.

\begin{tabular}{lccc}
\hline & Category $a$ & Category $b$ & Category $c$ \\
\hline Experiment 1 & 16 & 13 & 19 \\
Experiment 2 & 18 & 15 & 18 \\
Experiment 3 & 13 & 13 & 20 \\
Experiment 4 & 18 & 17 & 18 \\
\hline
\end{tabular}

is incomplete. Therefore, for each sample, it either implies FOD $\Theta_{a b}$ is complete or supports FOD $\Theta_{a b}$ is incomplete. The simulation results of the four experiments are given in Table 3.

According to Table 3, the identification for FOD $\Theta_{a b}$ is divided into two situations: complete FOD and incomplete
TABLE 4: Confusion matrix for Experiment 1.

\begin{tabular}{lcc}
\hline & & Actual \\
& com_FOD & incom_FOD \\
\hline Identified & & \\
com_FOD & 29 & 1 \\
incom_FOD & 11 & 19 \\
\hline
\end{tabular}

FOD, simply denoted as com_FOD and incom_FOD. Based on the experiment results, four confusion matrixes are derived, shown in Tables 4-7. And the recall rate, precision rate, and the overall accuracy rate can be calculated, as shown in Table 8. As shown in the last column of Table 8, the fourth 
TABle 5: Confusion matrix for Experiment 2.

\begin{tabular}{lcc}
\hline & \multicolumn{3}{c}{ Actual } \\
& com_FOD & incom_FOD \\
\hline Identified & 33 & 2 \\
com_FOD & 7 & 18 \\
incom_FOD & & \\
\hline
\end{tabular}

TABle 6: Confusion matrix for Experiment 3.

\begin{tabular}{lcc}
\hline & \multicolumn{3}{c}{ Actual } \\
& com_FOD & incom_FOD \\
\hline Identified & & \\
com_FOD & 26 & 0 \\
incom_FOD & 14 & 20 \\
\hline
\end{tabular}

TABle 7: Confusion matrix for Experiment 4.

\begin{tabular}{lcc}
\hline & & Actual \\
& com_FOD & incom_FOD \\
\hline Identified & 35 & 2 \\
$\quad$ com_FOD & 5 & 18 \\
incom_FOD & & \\
\hline
\end{tabular}

experiment occupies the highest accuracy for the identification, which shows that the simultaneous consideration of the three criteria could lead to the best accuracy compared with just simply considering one criterion. As also can be found from Table 8, Criterion 2 has the best performance in accuracy among the three criteria. That is because it takes the correlativity and difference of evidence into account.

In order to give a clear comparison to the information of the results, Figures 5-7 graphically show the results of recall, precision, and accuracy rate, derived from different experiments. It is found that in every experiment the recall rate of incom_FOD is greater than that of com_FOD, which says for each actual situation there is a greater possibility of judging correctly for the samples out of the FOD. And precision rate of incom_FOD smaller than that of com_FOD means the identification result is more accurate compared with the incom_FOD.

Figure 7 shows that Experiment 4 which simultaneously considers the three criteria has the best performance in accuracy. There are several reasons for this result. For example, in identifying the complete FOD the condition is weakened which means three criteria do not need to be simultaneously satisfied. According to Table 8, the accuracy rate for Criterion 3 is lower compared with other experiments. So this condition eliminates some of Criterion 3 influence.

In a word, for a sample FOD can be identified with a high degree of accuracy. According to this, the proposed method is proved to be effective. Moreover, if all these three criteria can be satisfied, the system identified in incomplete frame of discernment is correct with $88 \%$ of the accuracy.

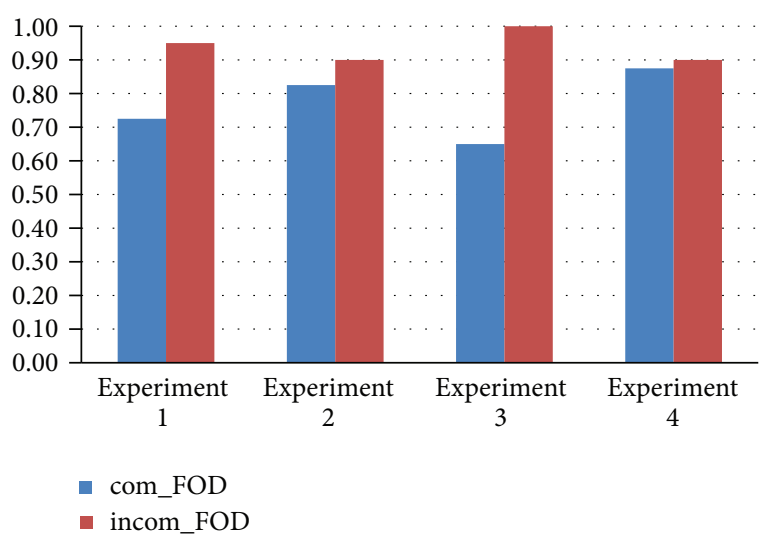

FIGURE 5: Recall rates of different situations in every experiment.

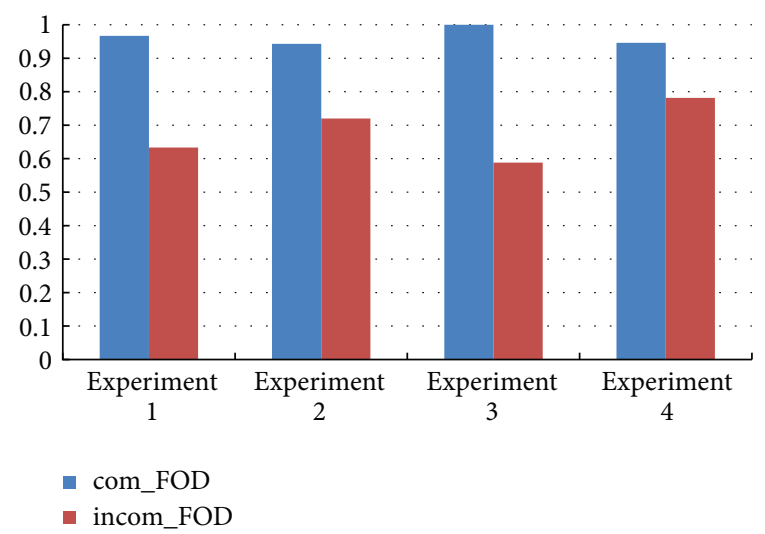

Figure 6: Precision rates of different situations in every experiment.

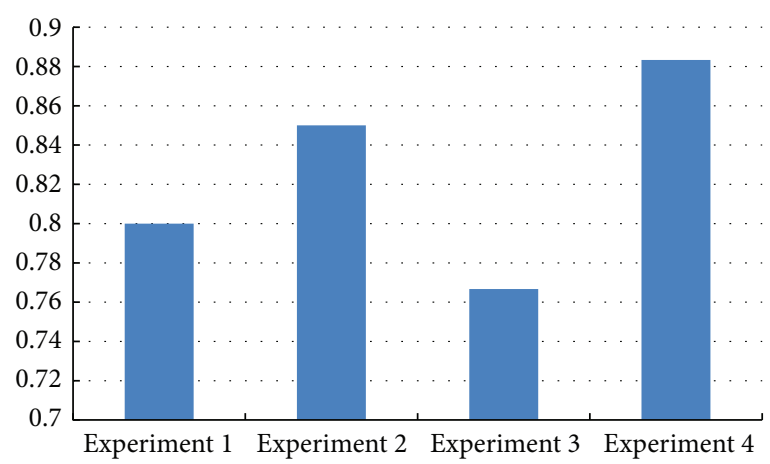

Figure 7: Accuracy rates of different situations in every experiment.

\section{Application}

In this section, the case of the motor rotor fault diagnosis is shown to demonstrate the effectiveness of the proposed method. In this case, we can obtain the rotor acceleration spectrum and the time domain vibration displacement average amplitude according to the sensor data. Then we can judge which kind of fault is the rotor based on in the proposed method.

There are three kinds of faults for the motor rotor: imbalance $(X)$, misalignment $(Y)$, and loose support base $(Z)$. We select the the frequency to be at an amplitude of 
TABLE 8: Performance of criteria in the experiments.

\begin{tabular}{lcccc}
\hline Experiment & Situation & Recall & Precision & Accuracy \\
\hline \multirow{2}{*}{ Experiment 1 } & com_FOD & 0.725 & 0.9667 & 0.63333 \\
& incom_FOD & 0.95 & 0.9429 \\
Experiment 2 & com_FOD & 0.825 & 0.72 & 0.85 \\
& incom_FOD & 0.9 & 1 & 0.7667 \\
Experiment 3 & com_FOD & 0.65 & 0.5882 & 0.9460 \\
Experiment 4 & incom_FOD & 1 & 0.7816 \\
\hline
\end{tabular}

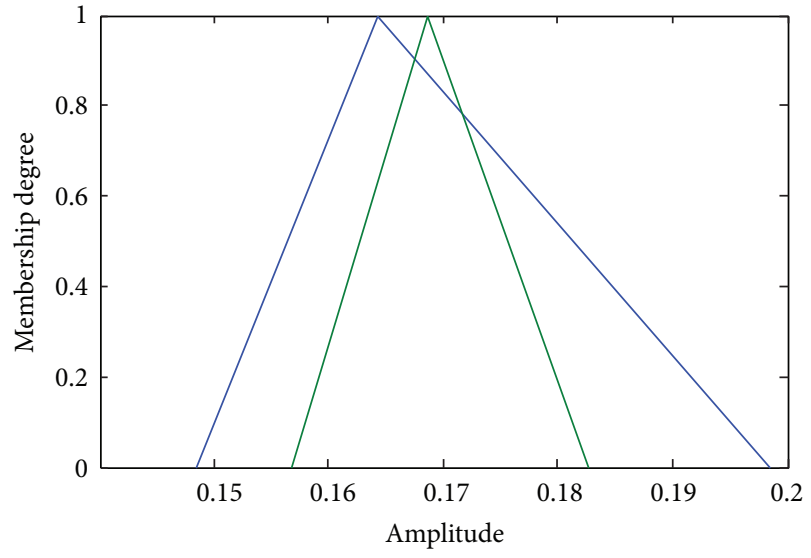

$-X$

$-Y$

(a) $1 X$

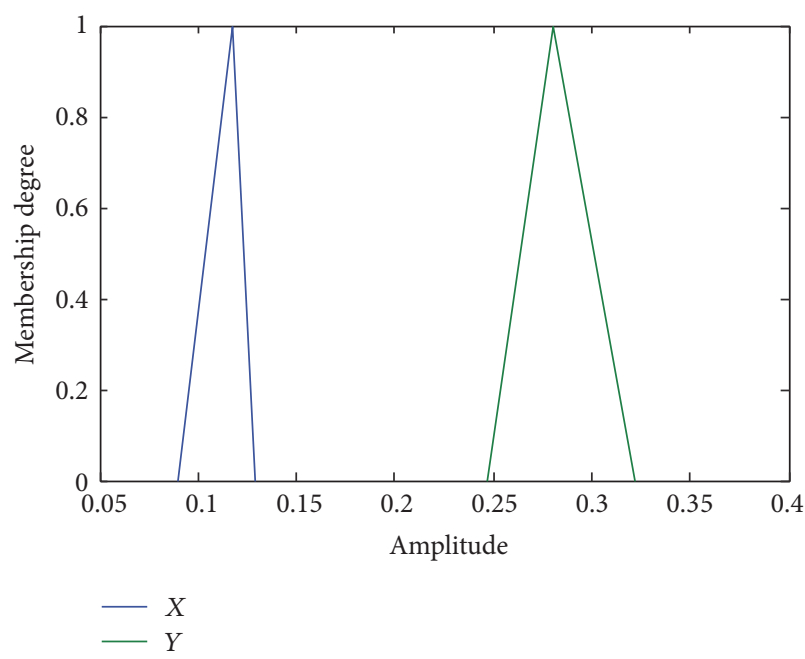

(c) $3 X$

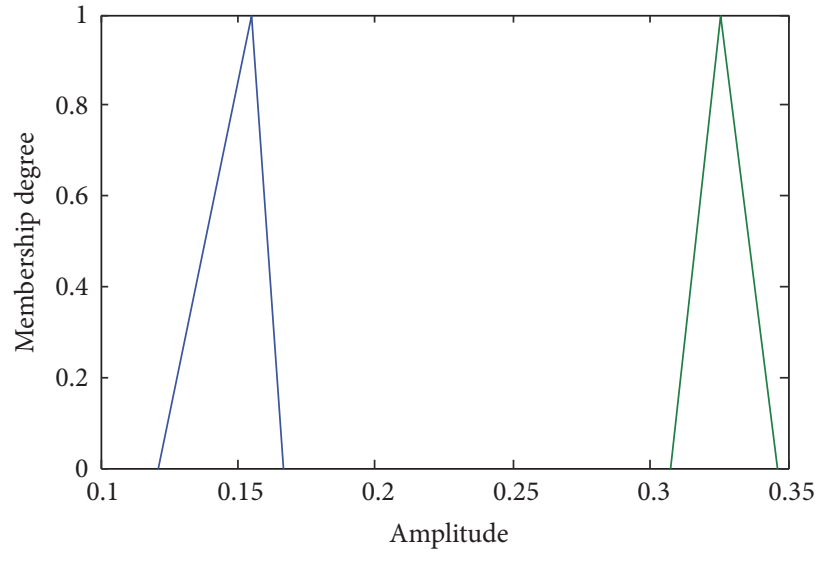

$X$

$-Y$

(b) $2 X$

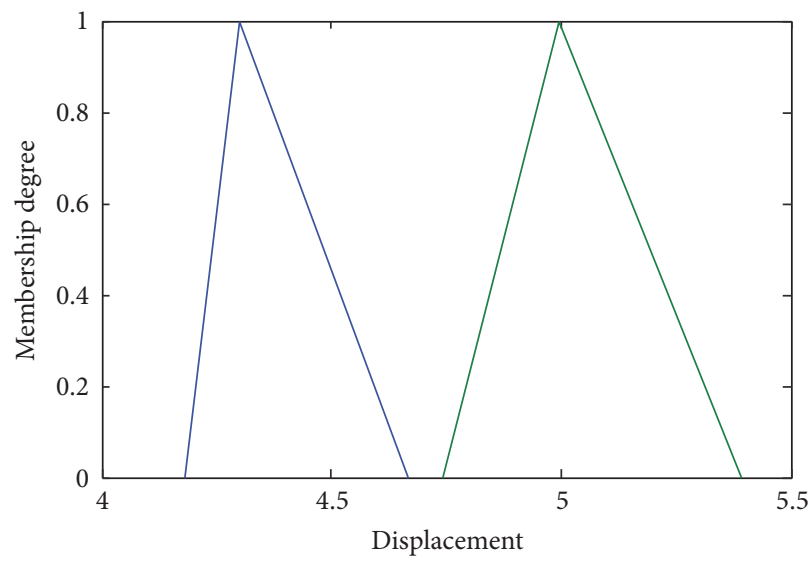

$-X$

FIGURE 8: Training models associated with four attributes of motor rotor fault.

vibration of $25 \mathrm{~Hz}(1 X), 50 \mathrm{~Hz}(2 X)$, and $75 \mathrm{~Hz}(3 X)$ and the vibration displacement average amplitude in time domain $(S)$ to be four $(q=4)$ attributions' eigenvalue. Each attribution eigenvalue was collected 40 times in the time interval $\Delta t=$ $16 \mathrm{~s}$ continuously, a total of 5 groups. These data can be divided into two parts: training set and test set. Training set also includes 90 samples, and test set includes the remaining 30 samples.

For each group, abstract the samples belonging to faults $X, Y$ to establish the incomplete FOD $\Theta_{X Y}=\{X, Y\}$. Based on the method mentioned in Section 4, the triangular membership function in Figure 8 and the GBPAs in Figures 9-11 


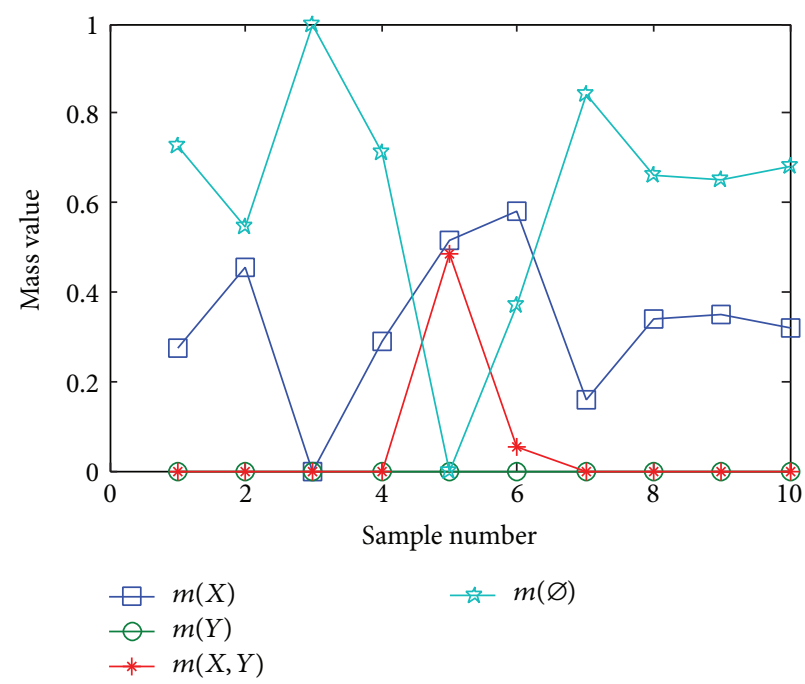

(a) $1 X$

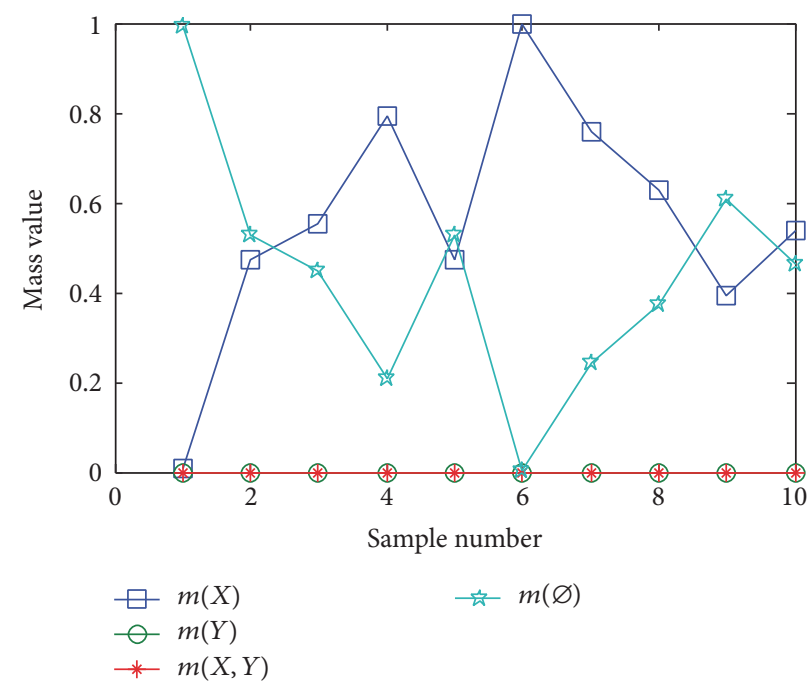

(c) $3 X$

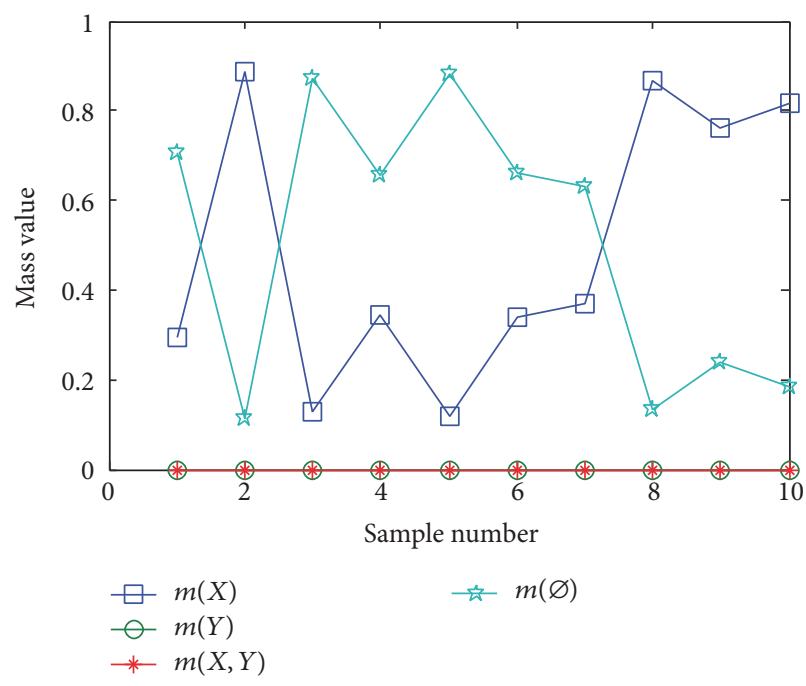

(b) $2 X$

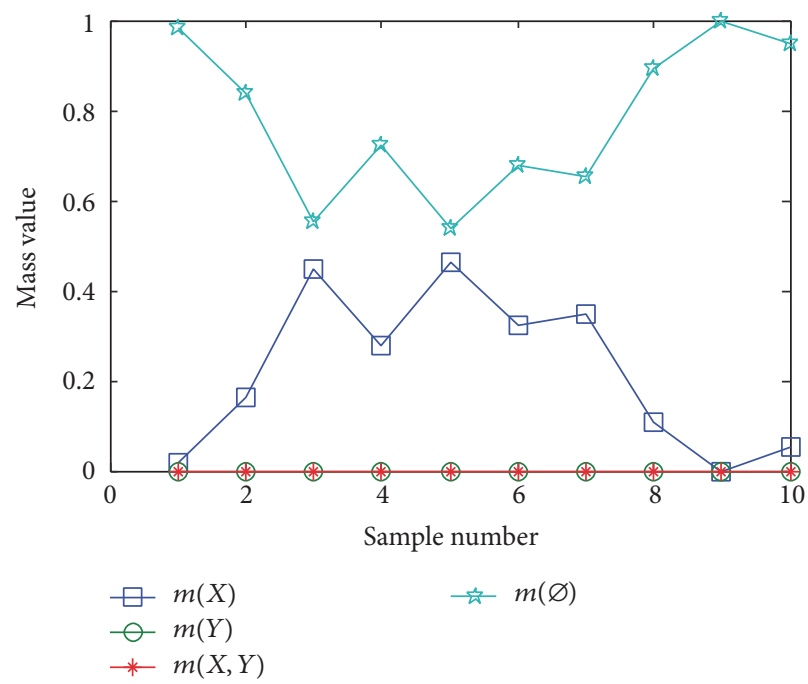

(d) $S$

FIgURE 9: Generated GBPAs associated with different attributes for the samples belonging to fault $X$ in the test set.

are generated. The same as the above experiments, there are four experiments taking into the criteria, respectively, and simultaneously. Then average identification results are shown in Figure 9, as well as the recall, precision, and accuracy rate based on the confusion matrix.

In Table 9, we can observe the same conclusion as the fourth experiment occupies the highest accuracy for the identification. Moreover for each experiment the accuracy is quiet high, and particularly all samples out of the FOD can be identified correctly in this application. The experiment results prove the effectiveness of the proposed method.

\section{Conclusions}

As stressed in previous studies, the method to identify the incomplete frame of discernment is still not presented.
And the incomplete framework is an important reason for the emergence of conflict. In this paper, a new method is proposed under the framework of GET, making full use of the available information contained in the generated pieces of evidence from a sample, to identify the incomplete frame of discernment. The case study of four experiments demonstrates the effectiveness of the proposed method. And in the experiments, three criteria parameters have a great influence on identifying the incomplete FOD.

The proposed method can be applied to many applications, such as fault identification and infectious disease surveillance. However, the incorrect identification result may be obtained because of the inaccuracy membership function. In the future, the proposed criteria of identifying incomplete FOD will be merged with the combination of highly conflicting pieces of evidence to obtain more reasonable combination result. 
TABLE 9: The average quantity of correct fault diagnosis in the experiments.

\begin{tabular}{|c|c|c|c|c|c|c|c|}
\hline Experiment & $X$ & $Y$ & $Z$ & Situation & Recall & Precision & Accuracy \\
\hline \multirow{2}{*}{ Experiment 1} & \multirow{2}{*}{5} & \multirow{2}{*}{6} & \multirow{2}{*}{10} & com_FOD & 0.55 & 1 & \multirow{2}{*}{0.70} \\
\hline & & & & incom_FOD & 1 & 0.53 & \\
\hline \multirow{2}{*}{ Experiment 2} & \multirow{2}{*}{8} & \multirow{2}{*}{7} & \multirow{2}{*}{10} & com_FOD & 0.75 & 1 & \multirow{2}{*}{0.83} \\
\hline & & & & incom_FOD & 1 & 0.57 & \\
\hline \multirow{2}{*}{ Experiment 3} & \multirow{2}{*}{7} & \multirow{2}{*}{7} & \multirow{2}{*}{10} & com_FOD & 0.70 & 1 & \multirow{2}{*}{0.80} \\
\hline & & & & incom_FOD & 1 & 0.625 & \\
\hline \multirow{2}{*}{ Experiment 4} & \multirow{2}{*}{8} & \multirow{2}{*}{8} & \multirow{2}{*}{10} & com_FOD & 0.80 & 1 & \multirow{2}{*}{0.87} \\
\hline & & & & incom_FOD & 1 & 0.71 & \\
\hline
\end{tabular}

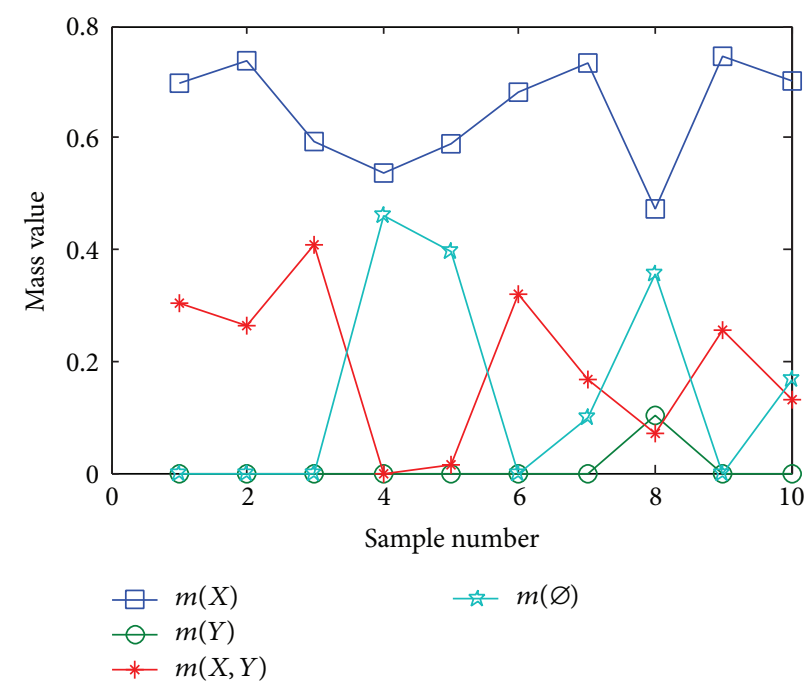

(a) $1 X$

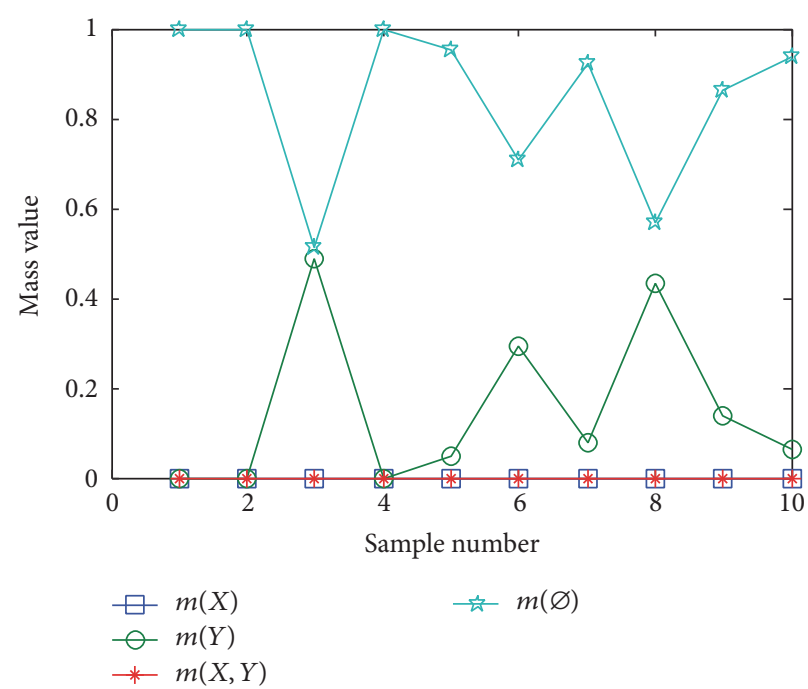

(c) $3 X$

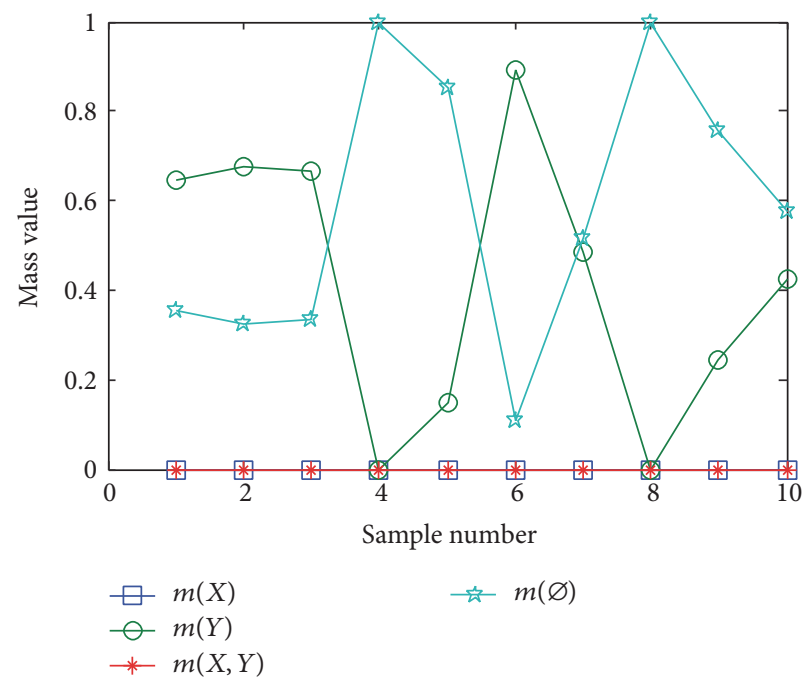

(b) $2 X$

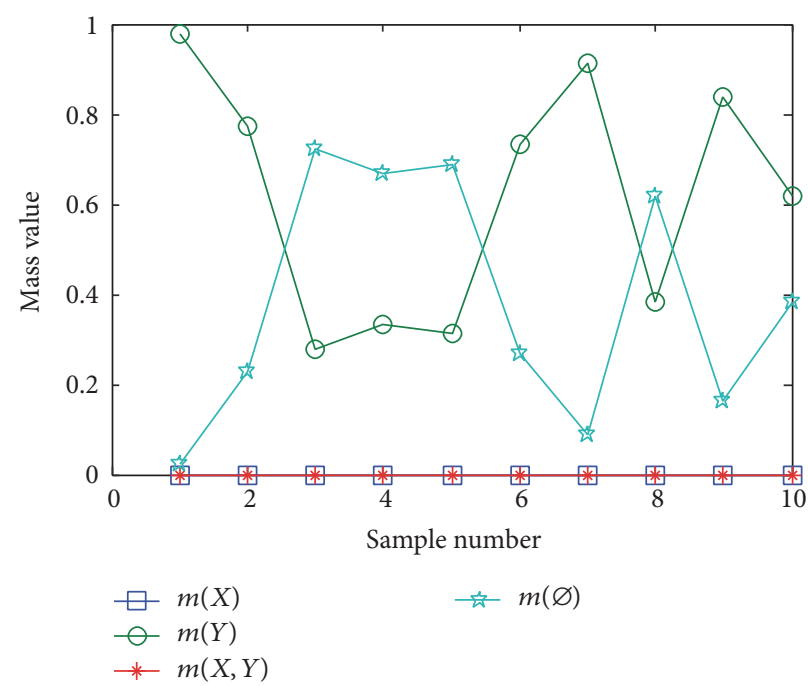

(d) $S$

FIGURE 10: Generated GBPAs associated with different attributes for the samples belonging to category $Y$ in the test set. 


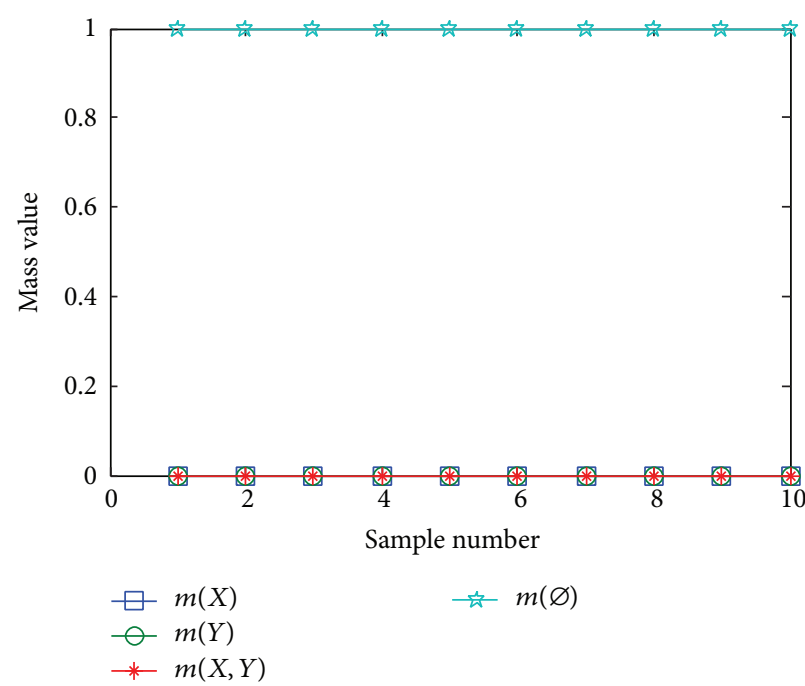

(a) $1 X$

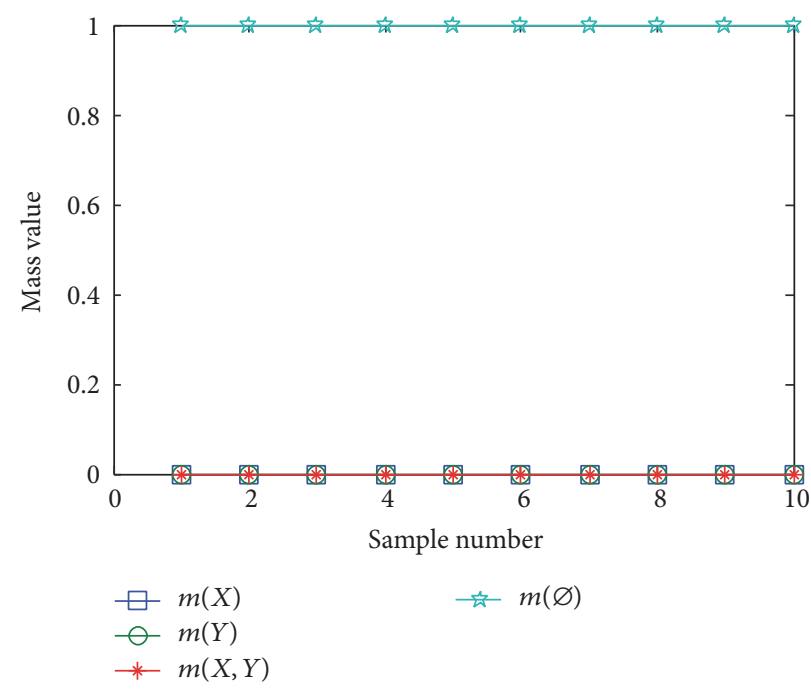

(c) $3 X$

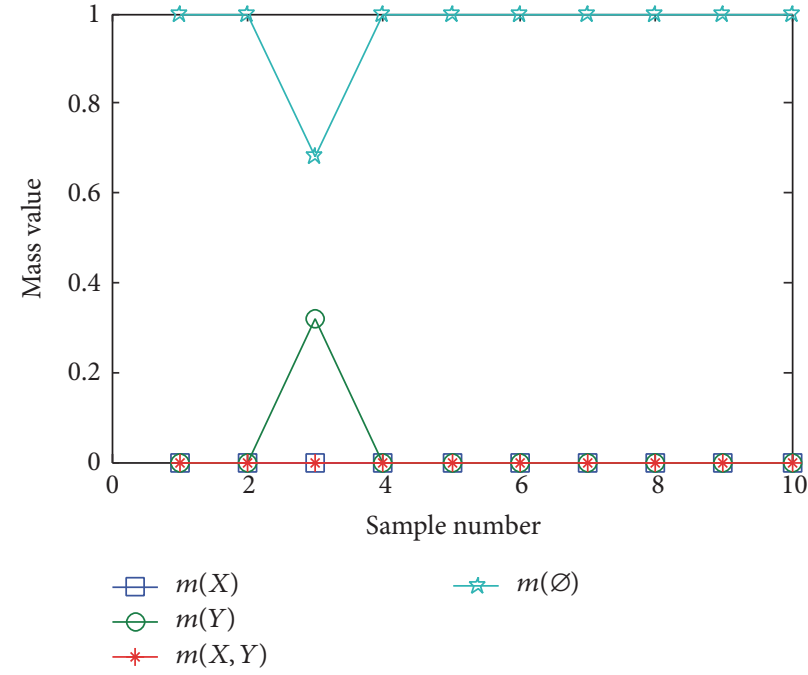

(b) $2 X$

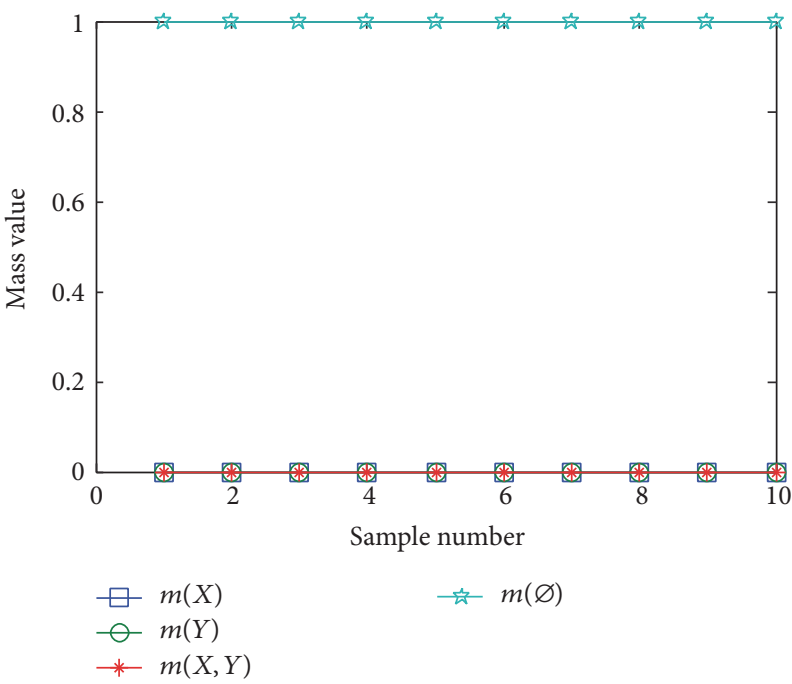

(d) $S$

FIGURE 11: Generated GBPAs associated with different attributes for the samples belonging to category $Z$ in the test set.

\section{Conflicts of Interest}

The authors declare that there are no conflicts of interest regarding the publication of this paper.

\section{Authors' Contributions}

Wen Jiang and Yue Chang conceived and designed the experiments. Yue Chang performed the experiments and wrote the paper. Shiyu Wang analyzed the data.

\section{Acknowledgments}

The work is partially supported by National Natural Science Foundation of China (Program nos. 61671384 and 61703338), Natural Science Basic Research Plan in Shaanxi Province of China (Program no. 2016JM6018), Project of Science and Technology Foundation, and Fundamental Research Funds for the Central Universities (Program no. 3102017OQD020).

\section{References}

[1] A. P. Dempster, "Upper and lower probabilities induced by a multivalued mapping," Annals of Mathematical Statistics, vol. 38, pp. 325-339, 1967.

[2] G. Shafer, A Mathematical Theory of Evidence, Princeton University Press, Princeton, NJ, USA, 1976.

[3] X. Deng and W. Jiang, "An evidential axiomatic design approach for decision making using the evaluation of belief structure satisfaction to uncertain target values," International Journal of Intelligent Systems, vol. 33, no. 1, pp. 15-32, 2018.

[4] X. Su, S. Mahadevan, W. Han, and Y. Deng, "Combining dependent bodies of evidence," Applied Intelligence, vol. 44, no. 3, pp. 634-644, 2016.

[5] W. Jiang, B. Wei, X. Liu, X. Li, and H. Zheng, "Intuitionistic fuzzy power aggregation operator based on entropy and its application in decision making," International Journal of Intelligent Systems, vol. 33, no. 1, pp. 49-67, 2018. 
[6] F. Xiao, "An Intelligent Complex Event Processing with D Numbers under Fuzzy Environment," Mathematical Problems in Engineering, vol. 2016, Article ID 3713518, 2016.

[7] C. Fu, J.-B. Yang, and S.-L. Yang, "A group evidential reasoning approach based on expert reliability," European Journal of Operational Research, vol. 246, no. 3, pp. 886-893, 2015.

[8] L. Chen, X. Zhou, F. Xiao, Y. Deng, and S. Mahadevan, "Evidential Analytic Hierarchy Process Dependence Assessment Methodology in Human Reliability Analysis," Nuclear Engineering and Technology, vol. 49, no. 1, pp. 123-133, 2017.

[9] W. Jiang and S. Wang, "An uncertainty measure for intervalvalued evidences," International Journal of Computers Communications \& Control, vol. 12, no. 5, pp. 631-644, 2017.

[10] X. Deng, F. Xiao, and Y. Deng, "An improved distance-based total uncertainty measure in belief function theory," Applied Intelligence, vol. 46, no. 4, pp. 898-915, 2017.

[11] T. Denœux, S. Sriboonchitta, and O. Kanjanatarakul, "Evidential clustering of large dissimilarity data," Knowledge-Based Systems, vol. 106, pp. 179-195, 2016.

[12] H. Zheng, Y. Deng, and Y. Hu, "Fuzzy evidential influence diagram and its evaluation algorithm," Knowledge-Based Systems, vol. 131, pp. 28-45, 2017.

[13] D. Han, W. Liu, J. Dezert, and Y. Yang, "A novel approach to pre-extracting support vectors based on the theory of belief functions," Knowledge-Based Systems, vol. 110, pp. 210-223, 2016.

[14] Y. Deng, "Deng entropy," Chaos, Solitons \& Fractals, vol. 91, pp. 549-553, 2016.

[15] X. Zhou, X. Deng, Y. Deng, and S. Mahadevan, "Dependence assessment in human reliability analysis based on D numbers and AHP," Nuclear Engineering and Design, vol. 313, pp. 243252, 2017.

[16] H.-C. Liu, Q.-L. Lin, and M.-L. Ren, "Fault diagnosis and cause analysis using fuzzy evidential reasoning approach and dynamic adaptive fuzzy Petri nets," Computers \& Industrial Engineering, vol. 66, no. 4, pp. 899-908, 2013.

[17] Z. Xu, C. Hu, F. Yang et al., "Data-driven inter-turn short circuit fault detection in induction machines," IEEE Access, vol. 5, no. 1, pp. 25055-25068, 2017.

[18] H.-C. Liu, L. Liu, Q.-L. Lin, and N. Liu, "Knowledge acquisition and representation using fuzzy evidential reasoning and dynamic adaptive fuzzy petri nets," IEEE Transactions on Cybernetics, vol. 43, no. 3, pp. 1059-1072, 2013.

[19] X. Wang, F. Yang, H. Wei, and L. Ji, "A risk assessment model of uncertainty system based on set-valued mapping," Journal of Intelligent \& Fuzzy Systems: Applications in Engineering and Technology, vol. 31, no. 6, pp. 3155-3162, 2016.

[20] X. Zhang, S. Mahadevan, and X. Deng, "Reliability analysis with linguistic data: An evidential network approach," Reliability Engineering \& System Safety, vol. 162, pp. 111-121, 2017.

[21] W. Jiang, C. Xie, M. Zhuang, and Y. Tang, "Failure mode and effects analysis based on a novel fuzzy evidential method," Applied Soft Computing, vol. 57, pp. 672-683, 2017.

[22] H.-C. Liu, J.-X. You, X.-J. Fan, and Q.-L. Lin, "Failure mode and effects analysis using D numbers and grey relational projection method," Expert Systems with Applications, vol. 41, no. 10, pp. 4670-4679, 2014.

[23] H.-C. Liu, L. Liu, and Q.-L. Lin, "Fuzzy failure mode and effects analysis using fuzzy evidential reasoning and belief rule-based methodology," IEEE Transactions on Reliability, vol. 62, no. 1 , pp. 23-36, 2013.
[24] Y. He, L. Hu, X. Guan, Y. Deng, and D. Han, "New method for measuring the degree of conflict among general basic probability assignments," Science China Information Sciences, vol. 55, no. 2, pp. 312-321, 2012.

[25] E. Lefevre, O. Colot, and P. Vannoorenberghe, "Belief function combination and conflict management," Information Fusion, vol. 3, no. 2, pp. 149-162, 2002.

[26] R. Haenni, “Are alternatives to Dempster's rule of combination real alternatives? Comments on "About the belief function combination and the conflict management problem" - Lefevre et al," Information Fusion, vol. 3, no. 3, pp. 237-239, 2002.

[27] C. K. Murphy, "Combining belief functions when evidence conflicts," Decision Support Systems, vol. 29, no. 1, pp. 1-9, 2000.

[28] D. Yong, S. WenKang, Z. ZhenFu, and L. Qi, "Combining belief functions based on distance of evidence," Decision Support Systems, vol. 38, no. 3, pp. 489-493, 2004.

[29] P. Smets and R. Kennes, "The transferable belief model," Artificial Intelligence, vol. 66, no. 2, pp. 191-234, 1994.

[30] W. Jiang and J. Zhan, "A modified combination rule in generalized evidence theory," Applied Intelligence, vol. 46, no. 3, pp. 630-640, 2017.

[31] C.-C. Chou, L.-J. Liu, S.-F. Huang, J.-M. Yih, and T.-C. Han, “An evaluation of airline service quality using the fuzzy weighted SERVQUAL method," Applied Soft Computing, vol. 11, no. 2, pp. 2117-2128, 2011.

[32] C. C. Chou, "A generalized similarity measure for fuzzy numbers," Journal of Intelligent \& Fuzzy Systems: Applications in Engineering and Technology, vol. 30, no. 2, pp. 1147-1155, 2016.

[33] C. Kahraman, S. C. Onar, and B. Oztaysi, "Fuzzy multicriteria decision-making: a literature review," International Journal of Computational Intelligence Systems, vol. 8, no. 4, pp. 637-666, 2015.

[34] X. Deng, D. Han, J. Dezert, Y. Deng, and Y. Shyr, "Evidence Combination from an Evolutionary Game Theory Perspective," IEEE Transactions on Cybernetics, vol. 46, no. 9, pp. 2070-2082, 2016.

[35] W. Jiang, A correlation coefficient of belief functions, https:// arxiv.org/abs/1612.05497.

[36] A. L. Jousselme, D. Grenier, and É. Bossé, "A new distance between two bodies of evidence," Information Fusion, vol. 2, no. 2, pp. 91-101, 2001.

[37] W. Jiang, J. Zhan, D. Zhou, and X. Li, "A method to determine generalized basic probability assignment in the open world," Mathematical Problems in Engineering, vol. 2016, Article ID 3878634, 11 pages, 2016.

[38] J. Zhang and Y. Deng, "A method to determine basic probability assignment in the open world and its application in data fusion and classification," Applied Intelligence, vol. 46, no. 4, pp. 934951, 2017.

[39] C. Sammut and G. I. Webb, Encyclopedia of Machine Learning, Springer Science \& Business Media, 2011. 


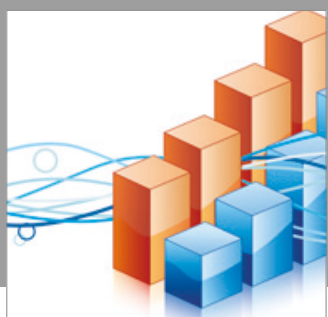

Advances in

Operations Research

vatersals

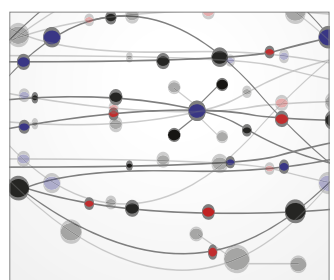

\section{The Scientific} World Journal
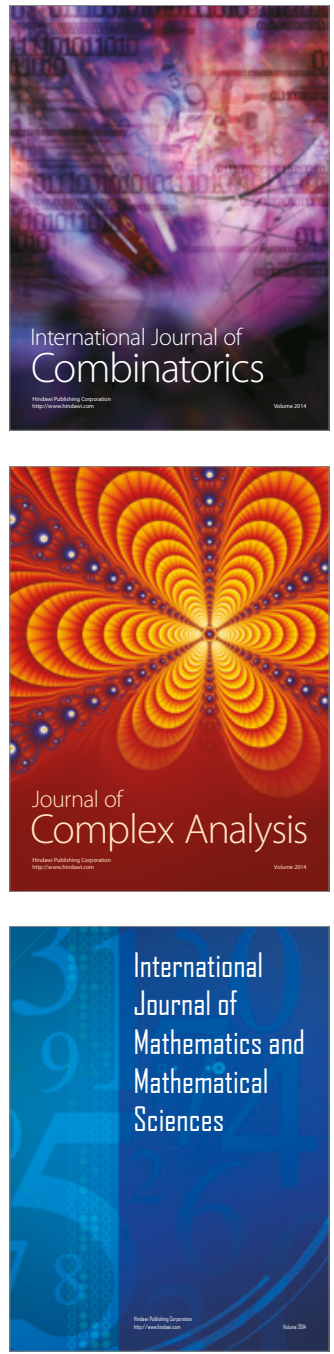
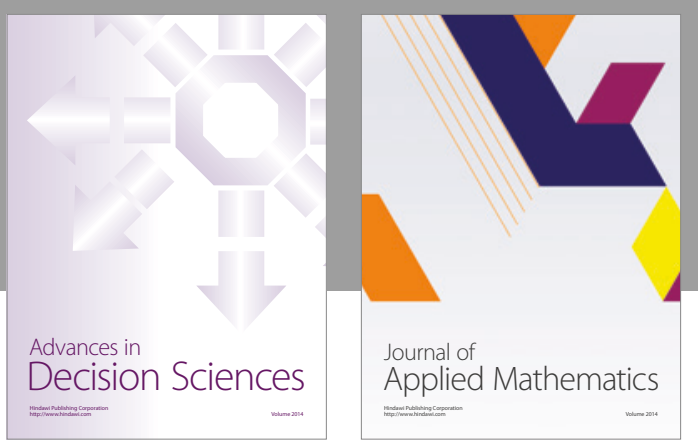

Algebra

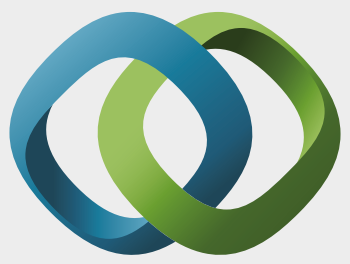

\section{Hindawi}

Submit your manuscripts at

https://www.hindawi.com
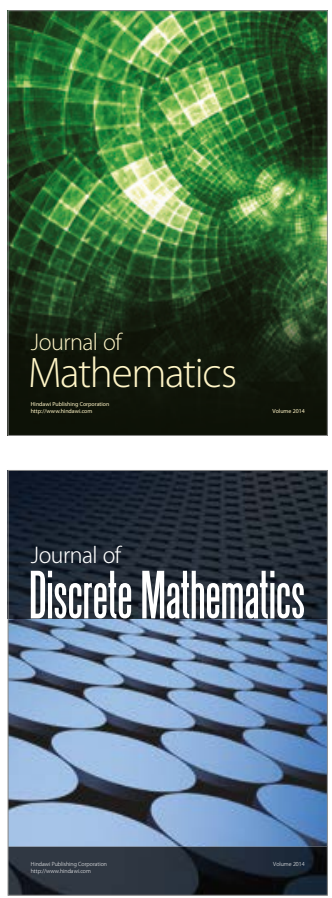

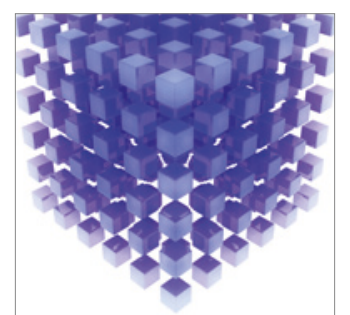

Mathematical Problems in Engineering
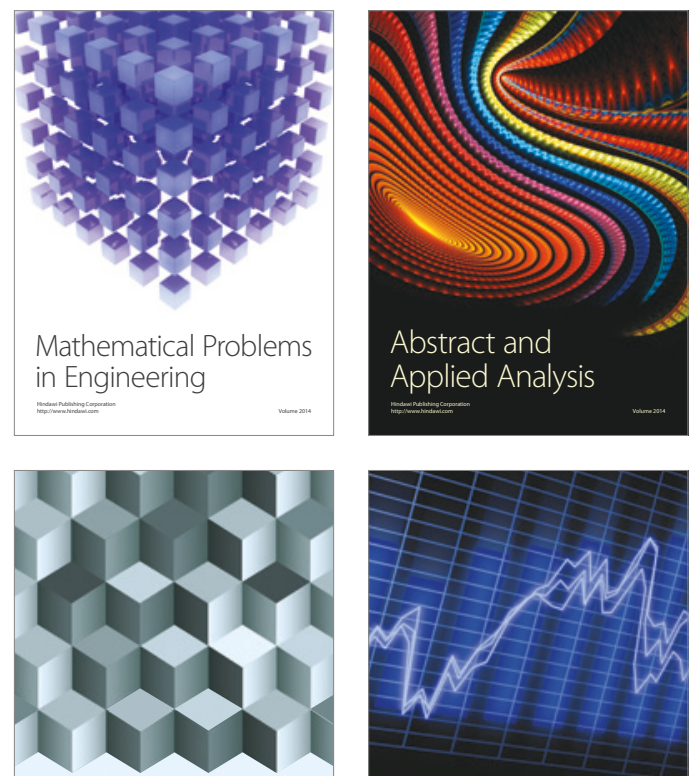

Journal of

Function Spaces

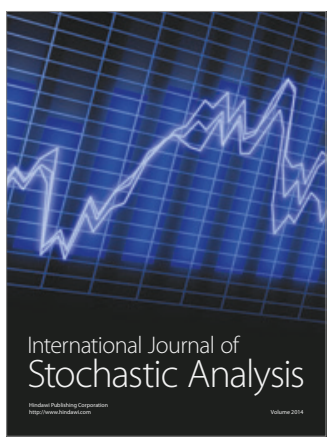

Probability and Statistics
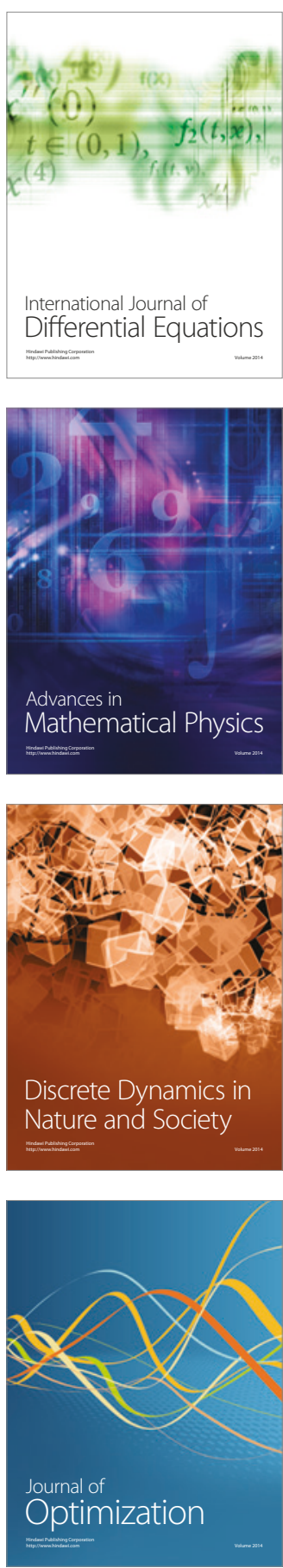